\title{
Open clusters in the Third Galactic Quadrant III. Alleged binary clusters ${ }^{\star}$
}

\author{
R. A. Vázquez ${ }^{1}$, A. Moitinho ${ }^{2}$, G. Carraro ${ }^{3, \star \star}$, and W. S. Dias ${ }^{4}$ \\ ${ }^{1}$ Facultad de Ciencias Astronómicas y Geofísicas de la UNLP, IALP-CONICET, Paseo del Bosque s/n 1900, La Plata, Argentina \\ e-mail: rvazquez@fcaglp.fcaglp.unlp.edu.ar \\ 2 SIM/IDL, Faculdade de Ciências da Universidade de Lisboa, Ed. C8, Campo Grande, 1749-016 Lisboa, Portugal \\ e-mail: andre@sim.ul.pt \\ 3 ESO, Alonso de Cordova 3107, Vitacura, Santiago, Chile \\ e-mail: gcarraro@eso.org \\ ${ }^{4}$ UNIFEI, Instituto de Ciências Exatas, Universidade Federal de Itajubá, Itajubá MG, Brazil \\ e-mail: wilton@unifei.edu.br
}

Received 23 December 2008 / Accepted 10 August 2009

ABSTRACT

\begin{abstract}
Aims. We aim to determine accurate distances and ages of eight open clusters in order to: (1) assess their possible binarity (2) provide probes to trace the structure of the Third Galactic Quadrant.

Methods. Cluster reddenings, distances, ages and metallicities are derived from ZAMS and isochrone fits in UBVRI photometric diagrams. Field contamination is reduced by restricting analysis to stars within the cluster limits derived from star counts. Further membership control is done by requiring that stars have consistent positions in several diagrams and by using published spectral types. Results. The derived distances, ages and metallicities have shown that none of the analysed clusters compose binary/double systems. Of the four candidate pairs, only NGC 2383/NGC 2384 are close to each other, but have different metallicities and ages. Ruprecht 72 and Ruprecht 158 are not clusters but fluctuations of the field stellar density. Haffner 18 is found to be the superposition of two stellar groups at different distances: Haffner 18(1) at $4.5 \mathrm{kpc}$ and Haffner 18(2) between 9.5 and $11.4 \mathrm{kpc}$ from the Sun. The derived distances and ages have been used to situate the clusters in the Galactic context. In particular, young stellar groups trace spiral structure at large Galactocentric radii. At least two clusters formed during the last few $10^{8} \mathrm{yr}$ in an interstellar medium with less than solar abundances. Conclusions. In contrast with the LMC, double clusters are apparently rare, or even non existent, in the undisturbed environment of the Third Galactic Quadrant. This leaves open the question of whether binary clusters form more easily toward denser and more violent regions of the Milky Way such as the inner Galaxy.
\end{abstract}

Key words. Galaxy: structure - open clusters and associations: general

\section{Introduction}

This paper continues the series of articles dedicated to analysing the homogeneous $U B V R I$ photometry of the fields of 30 open clusters presented in Moitinho (2001, hereafter Paper I). The programme has been motivated by our interest in investigating the structure of the Canis Major-Puppis-Vela region of the Galactic disc and its interconnection with large scale star formation.

The photometry described in Paper I, together with other observations collected by our group during the last decade, have resulted in new insights into the structure and stellar populations in the Third Galactic Quadrant (3GQ). Specifically, a new vision of the (stellar) spiral structure and warp has emerged in which it is seen that the Norma-Cygnus (or Outer) arm is defined over the whole 3GQ and that the Local (Orion) arm is not a small spur, but a large structure that reaches the warped outer disc (Carraro et al. 2005; Moitinho et al. 2006b; Vázquez et al. 2008). An unexpected repercussion of this scenario has been to

\footnotetext{
* The original photometry is only available in electronic form at CDS via anonymous ftp to cdsarc.u-strasbg. fr (130.79.128.5) or via

http://cdsweb.u-strasbg.fr/cgi-bin/qcat?J/A+A/511/A38

$\star \star$ on leave from Dipartimento di Astronomia, Università di Padova, Vicolo Osservatorio 2, 35122 Padova, Italy
}

cast considerable doubt on the existence of the highly publicised Canis Major dwarf galaxy (Martin et al. 2004). These results rely not only on the fundamental parameters derived for the clusters themselves, but also on careful analysis of the characteristics of the field population. From the sample described in Paper I, only NGC 2425, NGC 2635 (Moitinho et al. 2006a, Paper II of this series), NGC 2588 (Baume et al. 2004) and NGC 2401 (Baume et al. 2006) have been the subject of publications dedicated to their detailed photometric analysis and derivation of main physical parameters. In this paper we present a detailed analysis of the fields of four open candidate cluster pairs.

It is well known that stars tend to form in clusters rather than in isolation. The exact mechanisms that lead from the contraction of molecular clouds to star clusters are not well understood and there are possibly different physical paths (gravitational collapse; turbulence driven). One possible outcome, although apparently rare in the Galaxy, is the emergence of binary or double clusters (Ziyakhanovet al. 2004). From the theoretical point of view, the formation and evolution of binary clusters poses some challenges. Twenty years ago, it was clearly exposed by Efremov \& Sitnik (1988) that clusters tend to form part of associations which in turn form part of complexes and super-complexes of stars and gas. Therefore, similar chemical composition, age and dynamical behaviour are expected for binary clusters. Interestingly, $\mathrm{h}$ and $\chi$ Persei compose the only 
Table 1. Position of cluster centres and estimated radii.

\begin{tabular}{lccccc}
\hline \hline \multicolumn{1}{c}{ Cluster } & $\begin{array}{c}l \\
{\left[{ }^{\circ}\right]}\end{array}$ & $\begin{array}{c}b \\
{\left[{ }^{\circ}\right]}\end{array}$ & $\begin{array}{c}\alpha_{2000} \\
{[\mathrm{~h}: \mathrm{m}: \mathrm{s}]}\end{array}$ & $\begin{array}{c}\delta_{2000} \\
{\left[{ }^{\circ}{ }^{\prime}{ }^{\prime \prime}\right]}\end{array}$ & $\begin{array}{c}r \\
{\left[{ }^{\prime}\right]}\end{array}$ \\
\hline Cz 29 & 230.81 & +0.96 & $07: 28: 25$ & $-15: 24: 00$ & 1.50 \\
Haff 10 & 230.77 & +1.00 & $07: 28: 34$ & $-15: 21: 52$ & 1.65 \\
Haff 18 & 243.15 & +0.44 & $07: 52: 39$ & $-26: 23: 00$ & 3.90 \\
Haff 19 & 243.08 & +0.52 & $07: 52: 47$ & $-26: 17: 00$ & 3.30 \\
NGC 2383 & 235.27 & -2.45 & $07: 24: 43$ & $-20: 57: 00$ & 2.20 \\
NGC 2384 & 235.39 & -2.39 & $07: 25: 10$ & $-21: 01: 18$ & 1.75 \\
Rup 72 & 259.55 & +4.37 & $08: 52: 05$ & $-37: 36: 00$ & - \\
Rup 158 & 259.55 & +4.42 & $08: 52: 27$ & $-37: 34: 00$ & - \\
\hline
\end{tabular}

confirmed true double cluster known in our Galaxy. This is in contrast with the Large Magellanic Cloud (LMC), where about $10 \%$ of the known open clusters seem to belong to pairs and probably more than $50 \%$ of them are true binary clusters (Dieball \& Grebel 2000; Dieball et al. 2002). Following Bhatia (1990), the lifetimes of binary clusters depend on their mutual separation but also on the tidal force of the parental galaxy and on encounters with giant molecular clouds. The models of Bhatia (1990) indicate binary cluster lifetimes ranging from a few $10^{6} \mathrm{yr}$ to $4 \times 10^{7} \mathrm{yr}$. Besides pairs originating from the same star forming event (which we call "true binary/double clusters"), gravitational capture can also create cluster pairs. In this case, components can have different ages and compositions and will tend to eventually merge (de Oliveira et al. 2000).

The rest of this article is structured as follows: Sect. 2 describes the object sample and data set; Sect. 3 discusses the strategy for determining cluster parameters. Section 4 is dedicated to an analysis of each cluster. Section 5 contextualises the clusters in the Galaxy. Some final comments and conclusions are given in Sect. 6.

\section{Cluster sample}

The sample of 30 open clusters presented in Paper I was selected in the Galactic range $217^{\circ}<l<260^{\circ}$ and $-5^{\circ}<b<5^{\circ}$, with angular diameters of approximately $5^{\prime}$ and with estimated ages of less than $1.3 \times 10^{8} \mathrm{yr}(\log ($ age $) \sim 8.1)$. From that sample we found that Czernik $29(\mathrm{Cz} 29)$ and Haffner 10 (Haff 10); Haffner 18 (Haff 18) and Haffner 19 (Haff 19); NGC 2383 and NGC 2384 and Ruprecht 72 (Rup 72) and Ruprecht 158 (Rup $158)$ are potential members of cluster pairs.

Considering only the constraint of Galactic longitude, the open cluster catalogue of Dias et al. (2002) lists 7 candidate cluster pairs separated by less than $10^{\prime}$. Pairs filtered out by the selection criteria were Collinder 196/Collinder 198 having $b \sim 6.9^{\circ}$; Ruprecht 27/Alessi 18 because Ruprecht 27 is too old and Alessi 18 is a doubtful cluster appearing to be a random concentration in the sky; Ruprecht 52/Ruprecht 155 also appear to be random noise in the stellar distribution. This is also the case of Ruprecht 72/Ruprecht 158, although still with a slight possibility of being real entities and were thus kept. As will be seen later in Sect. 4.4, they are indeed fluctuations in the stellar distribution and not clusters. The observed cluster pairs are indicated in Table 1 that shows all, except one, of the optically visible double-cluster candidates in that region of the Milky Way.

At the time of the observations presented here (1994 and 1998), no all-sky infrared surveys were available. With the advent of 2MASS (Cutri et al. 2003), systematic searches for embedded star clusters became possible. A similar search to the one performed in the optical, but now over the infrared cluster catalogues of Bica et al. (2003) and Dutra et al. (2003), only reveals two more pairs. Another exercise than can be done is to search over the merged list of optically revealed and hidden clusters. In this case 6 further candidates arise. However, the occurrence of embedded/non-embedded physical pairs is unlikely given the different environments of the prospective components.

\subsection{Observations and reductions}

The observed fields superposed on the Digitized Sky Survey (DSS) are shown in Fig. 1. Observations and data reductions are extensively described in Paper I. Briefly, observations were acquired in two runs (1994 and 1998) at the CTIO 0.9m telescope. Both runs used the same Tek $2048 \times 2048$ CCD and the standard set of UBVRI filters available at CTIO. Typical seeing was about $1.2^{\prime \prime}$ although a few images presented higher values $\left(\approx 2^{\prime \prime}\right)$. The $0.39^{\prime \prime} /$ pixel plate scale resulted in a total field of view of $13^{\prime} \times 13^{\prime}$. Due to distortions (coma) in the the edges of the images, stars separated by less than 150 pix from the edges were not used, which limited the useful field of view to $11^{\prime} \times 11^{\prime}$.

Images were processed using IRAF routines for overscan, bias, and flat-field corrections. PSF photometry was performed using the IRAF/ DAOPHOT (Stetson 1987) package and calibrated with standard star measurements in Landolt (1992) fields. For further details see Paper I.

\section{Strategy for the derivation of cluster parameters}

\subsection{Cluster sizes}

The linear size is a fundamental quantity in a comprehensive characterisation of an open cluster. It is necessary to evaluate its evolutionary status through analysis of its density and other related parameters (see Aarseth 1996; de La Fuente Marcos 1997; Kroupa et al. 2001) such as the slope of the mass spectrum. It is also routinely used as criteria for separating cluster-dominated from field-dominated regions in photometric diagrams (Baume et al. 2004).

The most usual approach for estimating cluster sizes is to assume a centrally peaked spherical stellar density distribution and then to determine the the distance at which its radial density profile merges with the (flat) density of the stellar background. In practise, this determination is normally done either by visually setting the limit in a plot or by fitting King (1962) profiles to the density profile. However, both approaches assume a spherically symmetric distribution and, in the case of the King profiles, that the system is dynamically relaxed.

Because we are interested in determining the cluster radii to reduce field contamination in photometric diagrams, the following approach has been adopted: contour maps were computed in each field by choosing adequate kernel sizes and grid steps. Once a high and extended stellar density is identified, a circle enclosing its full range is drawn by eye. The cluster limit is taken as that defined by the radius of this circle. The cluster centre is considered to be the geometric centre of the cluster as opposed to the position of the density peak. The resulting contour maps of the four regions under study are shown in Fig. 2. Each panel in the figure also shows an insert indicating the number of stars per square arcminute. A clear example of the adequacy of the approach is Haffner 10 . Had we chosen the evident peak to compute the radial density profile, we would have largely overestimated the angular dimension of Haffner 10 and included many field stars. In the case of NGC 2384, which is identified as a concentration of a few bright stars, the density enhancement is irrelevant, suggesting either that the cluster does 
R. A. Vázquez et al.: Binary clusters
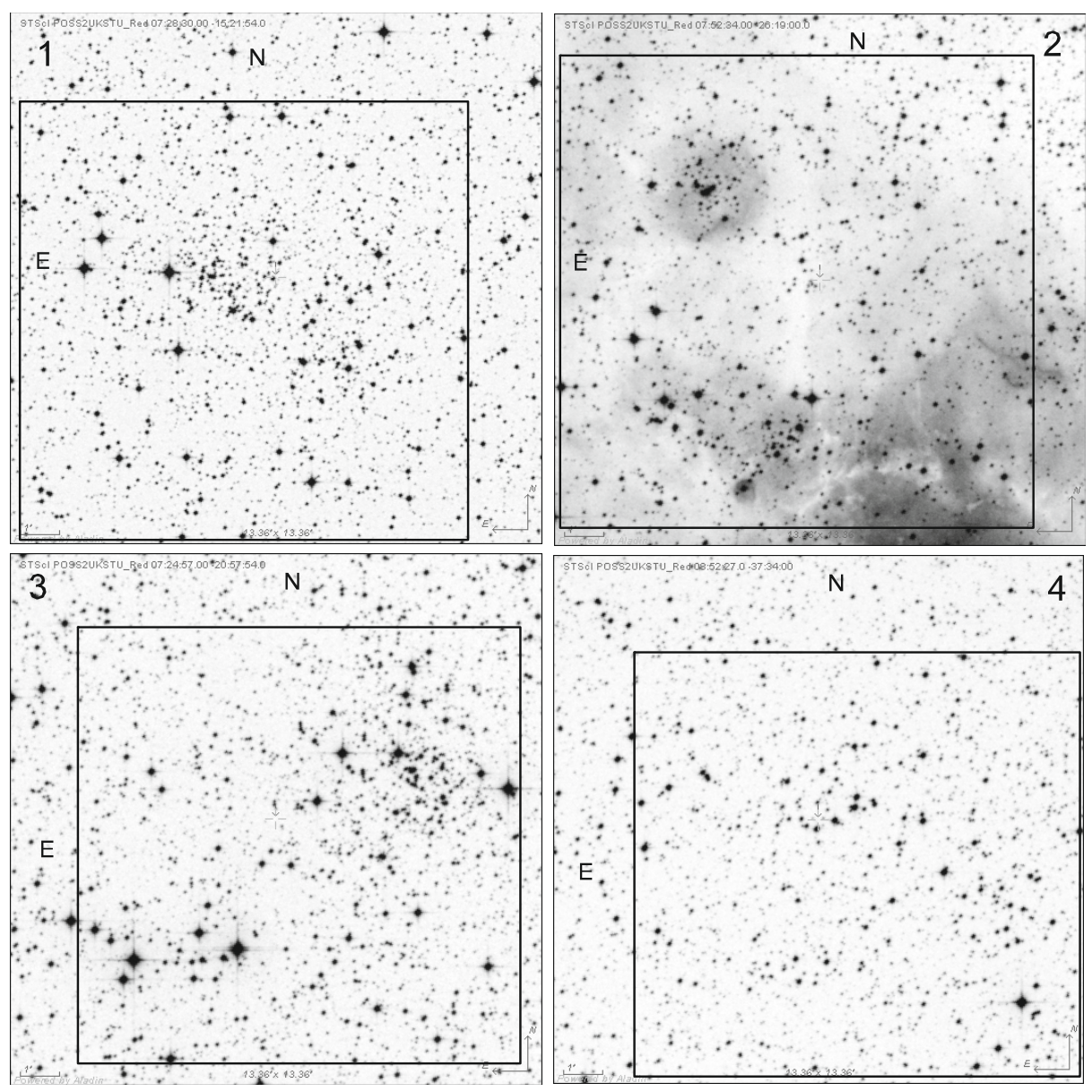

Fig. 1. DSS images of the four regions investigated in this article. The squares delimit the areas covered by the photometric measurement (East is at left and North at top): 1) Czernik 29 and Haffner 10;2) Haffner 18 and Haffner 19; 3) NGC 2383 and NGC 2384; 4) Ruprecht 72 and Ruprecht 158.
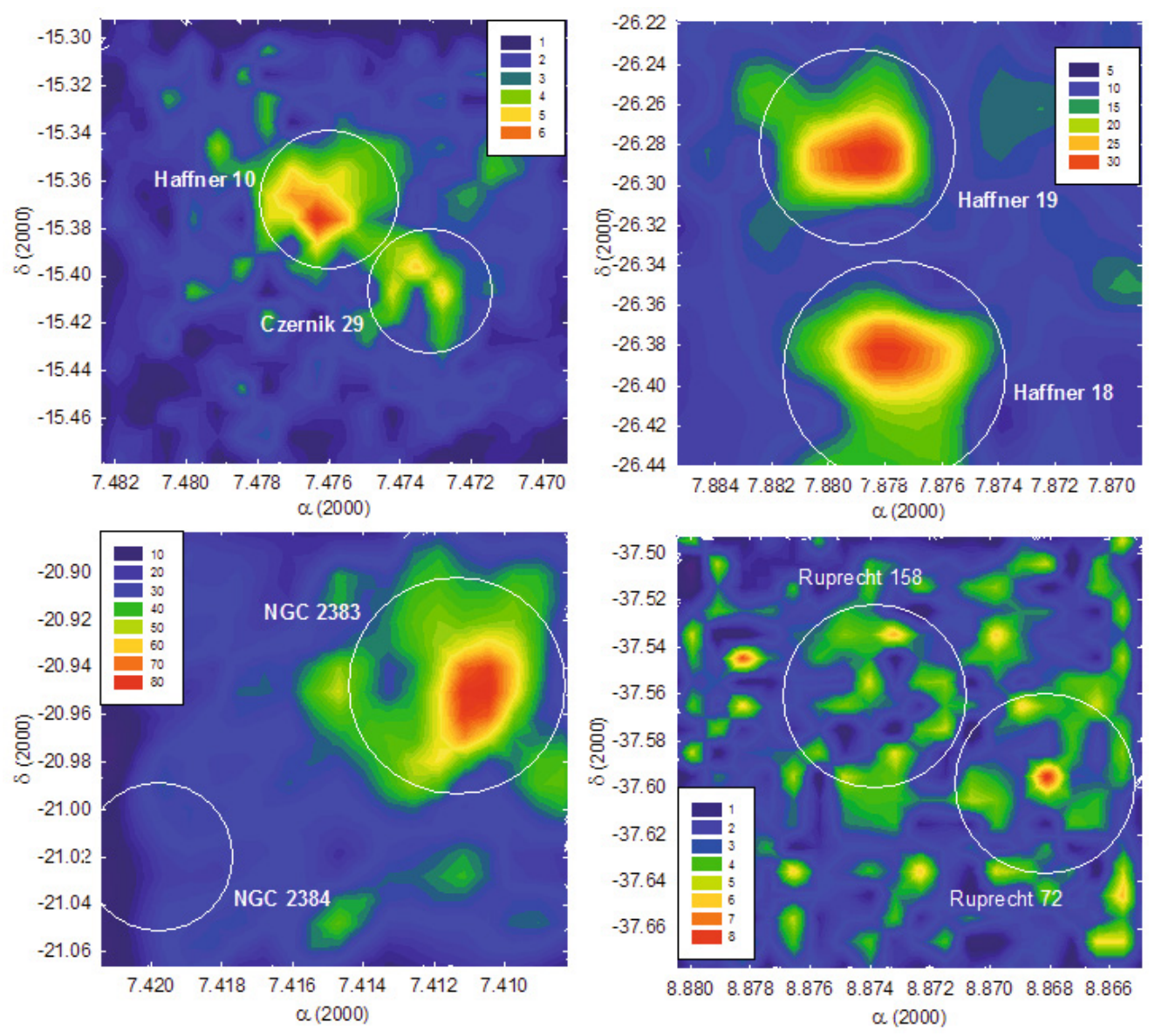

Fig. 2. Contour plots derived in each frame. The density scale - star number per square arcminute - is shown in the respective inserts. Circles are adopted cluster boundaries. North is at the top and East to the left. 
not exist or that it lacks faint stars. Here the cluster is assumed to be within a circle comprising the bright stars. As for the field of Ruprecht 72 and 158, no density enhancements or obvious conglomerates are seen. This field will be further discussed in Sect. 4.4. Table 1 gives the new cluster centres in galactic longitude, latitude, $\alpha_{2000}, \delta_{2000}$ and the adopted radii. In the cases of Ruprecht 72 and Ruprecht 158, centres and radii are those from the Dias et al. (2002) catalogue.

\subsection{Cluster membership}

Except for Ruprecht 72 and 158 (see Table 1) the regions included in this study are not far from the formal Galactic plane $b=0^{\circ}$. Under this circumstance, interstellar absorption and field interlopers may make membership assignment of faint stars a difficult task. Although an extensive discussion of this can be found in Vázquez et al. (2008), here we outline a few essential points: First, cluster parameters are determined using only stars within the determined cluster limits. This eliminates avoidable field contamination, enhancing the contrast between cluster and field distributions in photometric diagrams. Second, the location of each star is checked for consistency in different photometric diagrams, taking into account consistent reddening solutions, up to the magnitude limits where they become immersed in the field star distribution.

Third, for young clusters, the $V$ vs. $U-B$ diagram can be particularly useful for eliminating most A-F type foreground stars that usually contaminate the vertical part (early type) of cluster sequences in the $V / B-V$ plane. This is due to the greater sensitivity of the $U-B$ index to changes in stellar temperature compared to $B-V$ (Meynet et al. 1993). Further membership control is done when spectral types are available. Nevertheless, membership assignments for stars later than $A 0$ may remain uncertain. However, except for distance, determining the other basic cluster parameters, reddening and age, does not require identification of the fainter members.

\subsection{Reddenings, distances and ages of the clusters}

Mean colour excesses, $E_{(B-V)}$ and $E_{(U-B)}$, were computed in each cluster through the zero age main sequence (ZAMS) fitting method. The position along the reddening-free ZAMS of several spectral types earlier than $\mathrm{A} 0$ is indicated to help visual inspection of the two-colour diagram (TCD). The solar content ( $Z=0.019$ ) Schmidt-Kaler (1982) ZAMS (hereinafter SKZ) has been shifted along the reddening line given by the standard relation $E_{(U-B)}=0.72 \times E_{(B-V)}+0.05 \times E_{(B-V)}^{2}$ to fit the cluster sequence. The $E_{(B-V)}$ uncertainties quoted in Table 4 (in advance) reflects the range of ZAMS displacement to bracket cluster members. In a few cases ( $\mathrm{Cz} 29$, Haffner 10 and NGC 2383) the SKZ line does not follow the shape of the cluster sequence beyond the location of A-type stars (the ZAMS knee). Since the morphology of the ZAMS in this portion of the TCD strongly depends on the metal content, we made several trials with intrinsic colour-colour lines of different metallicities (Girardi et al. (2000) -hereafter G00) to find the interval that best approaches the cluster sequence shape. A mean value of the metal content was then assumed and also of $E_{(B-V)}$-with an acceptable level of departure- representing the uncertainty of these parameters (as shown in Table 4).

Wherever solar metal content is assumed, cluster apparent distance moduli were visually derived by fitting the SKZ to the observed cluster main sequence (MS) in colour-magnitude diagrams (CMDs). As said above, a different from solar metal content may become evident in some clusters from the reddening estimation. Therefore, we applied the same metal content set of isochronous lines (Girardi et al. 2000) until the best MS match is achieved in the CMD. The method is exemplified with the cluster Haffner 10 (see Sect. 4.1.1) where a simultaneous fit of the "turn-off", the Red Clump and MS was performed using the appropriate isochrone. Notice that in cases like this one, we can simultaneously obtain the color excess, the distance modulus and also the age of the cluster. In one case, given the large amount of internal reddening (see Sect. 4.2.1), the range of distance modulus is so large that we only indicate extreme values of the interval instead of the fitting uncertainty. Regardless of the metal content adopted, the fitting error of the apparent distance modulus for each cluster was estimated by eye as discussed below.

Cluster ages were derived by the same technique of fitting the isochrone (Girardi et al. 2000) set that reasonably matches the shape of the observed cluster sequence regarding the adopted metal content used to compute color excesses and distance moduli. Despite the special care put in fitting all the potential cluster members, some sequences are not satisfactorily reproduced by one single isochrone, as said above, and a range of them has been indicated in each cluster of Table 4 . Known reasons to fail the fitting include the effect of un-resolved binaries, stellar rotation and variable reddening due to remaining material from the star formation process (in very young clusters) that broaden the cluster sequence. This point is crucial since age estimates may vary strongly (up to $100 \%$ for those younger than $10^{7}$ years) if the effects mentioned above are not conveniently accounted for. If potential PMS-stars were detected in a cluster, we compared the estimated nuclear age to the one derived by fitting pre-main sequence isochrones from Siess et al. (2000) assuming a metal content similar to that adopted to compute the nuclear age.

The removal of interstellar absorption in a cluster, $A_{V}$, allows one to obtain the true distance modulus. $A_{V}$ has been determined from the above colour excess estimate via the ratio of total to selective absorption, $R=A_{V} / E_{(B-V)}$. Though analysis in Paper I has shown that in this part of the Galaxy a normal value of $R=$ 3.1 is to be expected, an independent check of the reddening law was performed by examining the field stellar distribution in plots such as the one in Fig. 4. For guidance we show in each $B-V / V-$ $I$ planes the slopes of the reddening vectors for $E_{(V-I)} / E_{(B-V)}=$ 1.244 - normal reddening law,$- R=3.1$, (Dean et al. 1978) and $E_{(V-I)} / E_{(B-V)}=1.5$ for $R=3.75$ together with the intrinsic relation for dwarf and giant stars from Cousins (1978).

Our strategy of visually fitting the SKZ (or other intrinsic lines) to the observed cluster sequence has been extensively treated in the literature; see, e.g., Blaauw (1963), Phelps \& Janes (1994) and Kassis et al. (1997), for a quantitative approach to the many factors (photometric errors, binaries, double stars, metal content, etc.) that reduce the accuracy of the parameters in a typical cluster. Aware of these effects, we decided that the best way to minimize their influence and to obtain a reasonably good parameter estimation of a cluster is by fitting the ZAMS to the remaining envelope of the MS (as stated by Turner 1973 and Turner 1976). The same holds for the isochrone fitting in the next subsection.

\section{Analysis}

This section presents the photometric analysis of each cluster field. An error cut-off of 0.10 mag was applied to all data plotted 


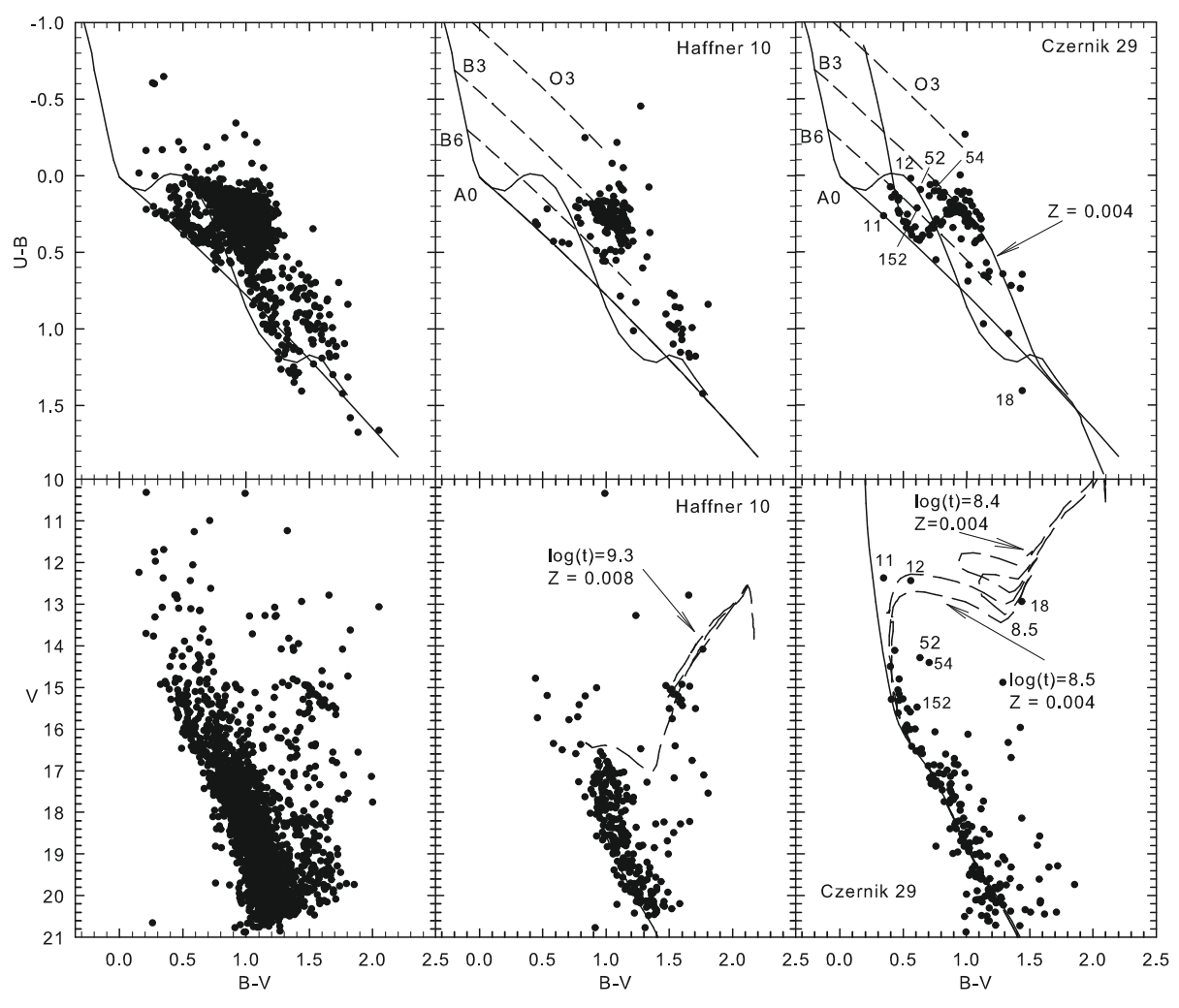

Fig. 3. Upper left panel: the TCDs for the entire region. Middle upper panel: Haffner 10 area. Right upper panel: Czernik 29. The SKZ (solid line) is shown together with the colour excess relation for O3-, B3-, B6- (dashed lines) and A0-type stars (solid). The reference intrinsic fitting line from G00 to Czernik 29 sequence, for $Z=0.004$, is discussed in the text. Lower panels: the correspondent CMDs where long-dashed lines are the isochrone [ $\log (\mathrm{t})]$ fits from G00 to Haffner 10 and Czernik $29 \mathrm{CMD}$ sequences, for $Z=0.008$ and $Z=0.004$, respectively as discussed in the text. Some stars, pointed out using our notation, are also discussed in the text. in the photometric diagrams. This is done to minimise artifacts introduced by high noise and low completeness.

\subsection{Haffner 10 and Czernik 29}

These two objects, located in Puppis, were the subject of an early $U B V$ photographic study by FitzGerald \& Moffat (1980) who concluded that they form a physical pair of relatively young objects at $2.9 \mathrm{kpc}$. Pietrukowicz et al. (2006) published a BVI CCD study of these two clusters together with NGC 2425. They derived no reddening, distance or age for Czernik 29 and suggested that it is not related to Haffner 10, which they determined to be a 2.5 Gyr cluster at $3.1-4.3 \mathrm{kpc}$.

Figure 3 shows the TCD and CMD (upper and lower left panels respectively) of the CCD field including both clusters. The TCD looks peculiar as at least three star sequences are seen: first, a hook-like structure appears at $0.1<U-B<0.5$ and $0.7<$ $B-V$; second, a dense clump of stars at $0.0<U-B<0.5$ and $0.5<B-V<1.2$ that accumulate below $V>16$ and is followed by a tail probably composed of giants and/or subgiants/dwarfs. A third sequence appears at $B-V>1.3$ which is mostly composed of field giants. Only a few bright hot stars appear, spread out in the field.

The diagrams become simpler when only stars within cluster limits (Table 1) are plotted.

\subsubsection{Haffner 10}

The TCD of Haffner 10 (Fig. 3, middle upper panel) displays an absence of late type dwarfs. The well separated concentration of late type stars corresponds to the red clump and a few field giants, as supported by inspection of the the $V / B-V$ CMD (Fig. 3, middle lower panel). The presence of the cluster is clearly seen in this CMD as well as in the $V / V-I$ CMD (Fig. 4,upper panel), showing a clear main sequence, turn-off (TO) and red clump.

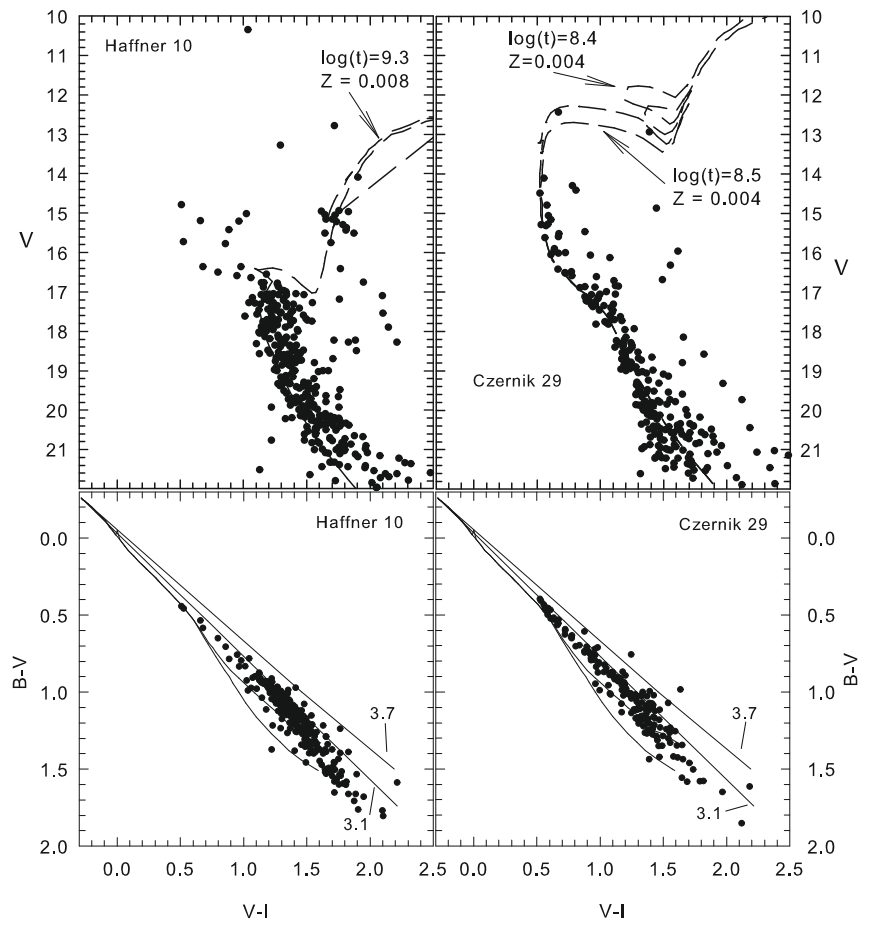

Fig. 4. Upper panels: the $V / V-I$ CMD for Haffner 10 (left) and Czernik 29 (right). Dashed lines are the isochrone [log (t)] fitting from G00 at the indicated metal content Z. Lower panels: the corresponding $B-$ $V / V-I$ TCDs. Straight lines show the colour excess relation for $R=$ 3.1 and 3.7 (Dean et al. 1978). The curved lines are the mean intrinsic colours for stars of Luminosity Class V and III from Cousins (1978).

There is a number of blue stars stars above the TO which could qualify as blue-straggler candidates. However, Haffner 10 as well as Czernik 29 are seen in projection against the Local arm 

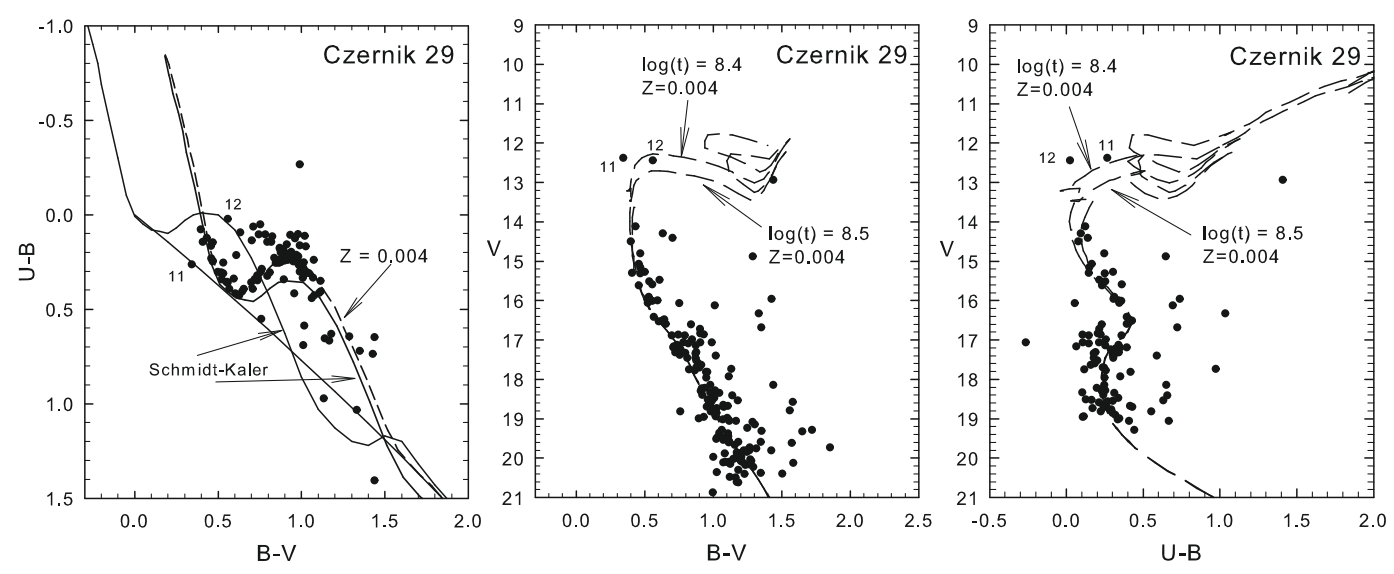

Fig. 5. Left: enlargement of the TCD of Czernik 29 in Fig. 3. The fitting of the SKZ (solid line) and the mean intrinsic curve for $Z=0.004$ (short-dashed line) from G00 are indicated. The colour excess relation for A0-type stars is also shown. Middle: the same in the V/B-V CMD; the two best isochrones $[\log (\mathrm{t})]$ fittings at $Z=0.004$ are pointed out. Right: same as in middle in the $V / U-B \mathrm{CMD}$

and to the less populated (in this region of the Galaxy) Perseus arm (Moitinho et al. 2006a; Vázquez et al. 2008). The extensive discussion in Carraro et al. (2008) has shown that under these circumstances some blue-straggler candidates are likely young field A-type stars not far from their birthplaces in the spiral arms. As will be seen later on, both clusters are at almost the same distance as the Local and Perseus arms in this this direction.

The extinction law toward Haffner 10 is checked in the $B-$ $V$ and $V-I$ diagram of Fig. 4 (left lower panel). The stellar distribution is consistent with a normal extinction law, $R=3.1$, supporting the results from Paper I.

A simple quick inspection of the TCDs and CMDs of Haffner 10 shows that it is an old cluster. In the absence of early type stars to constrain reddening alone, the cluster reddening, distance and age were determined by finding the isochrones and parameters that best fit simultaneously the main sequence, TO and the red-clump in different CMDs. The best match was found for a mean age of $2.5 \mathrm{Gyr}(\log (t)=9.2-9.4)$ with metal content $Z=0.008 \pm 0.001$ (the same one found by Pietrukowicz et al. (2006)), reddened by $E_{(B-V)}=0.55 \pm 0.03$ and with a distance modulus $V-M_{V}=14.55 \pm 0.25$. This locates Haffner 10 at $d=3.7 \pm 0.4 \mathrm{kpc}$ from the Sun, in agreement with Pietrukowicz et al. (2006).

Finally, we discuss the apparent thickness of the main sequence seen in both CMDs. Indeed, the strip occupied from the MS to about 1 mag brighter (or 0.2 mag bluer) is completely populated by stars. The immediate explanation would be field star contamination, since field stars are expected to be present in the photometric diagrams and in this case there are not many found outside the strip. However, both, the blue and red envelopes of the strip have the same curved shape, and do not define the typical triangular/wedge shape found for most fields star distributions.

This same strip morphology is also seen in the data of Pietrukowicz et al. (2006) covering a smaller area, so it is not likely be a selection artifact. It is likely due to one of two factors, or a combination of both: a) Haffner 10 is the remainder of an initially well populated cluster with many binary stars. b) the cluster is seen projected against a stellar field with similar properties of reddening and distance. Although there are undoubtedly binaries in the cluster, we are inclined to consider the second possibility. If binaries were to dominate, then why no/few MS field stars are observed would be very hard to justify. If there was an originally very populated cluster, we would see significant signatures of it over larger areas, which we do not.

Under the similar-field scenario, we notice that:

a) the maps of Moitinho et al. (2006a), Vázquez et al. (2008) and Fig. 15 in Sect. 5 situate Haffner 10 on the far side of the Local arm or the inner side of Perseus (both arms cross at about this Galactic longitude). Approximately $1 \mathrm{kpc}$ after Haffner 10, there is a complex of CO clouds which can block most background stellar contamination, thus leaving the blue/faint side of the MS unpopulated. This would cut the left side of the typical field triangle/wedge.

b) As for the bright/red side, which is the populated side, both the shape (MS like) and thickness ( $\sim 1$ in the magnitude axis) mag are compatible with a stellar distribution affected by a similar amount of reddening as is Haffner 10 and distributed from the distance to the cluster to about $1 \mathrm{kpc}$ closer. Given that the location of Haffner 10 and that the Local arm is about $1 \mathrm{kpc}$ wide, this is the expected distribution of Local arm stars in the CMDs.

\subsubsection{Czernik 29}

The field of Czernik 29 (upper and lower right panels in Fig. 3) is simpler to interpret. Since field contamination is not strong, the cluster sequence is easily distinguishable, slightly reddened and composed of late B- and A-type stars. The $V / V-I$ CMD (right upper panel of Fig. 4) exhibits a well defined MS down to $V=19$. The two brightest blue stars (11 and 12), as well as the others labelled 52, 54 and 152 in Fig. 3, are not cluster members but foreground stars given their locations in the CMD and TCD.

The TCD in Fig. 5 of Czernik 29 shows two tentative ZAMS fits to the cluster sequence. On one side, the shape of the SKZ (shifted solid line) does not fit the whole sequence. On the other, it is well fitted by the metal poor $Z=0.004 \pm 0.002$ reference curve (short-dashed line). Therefore, adopting this value, the fit yields colour excesses $E_{(B-V)}=0.47 \pm 0.02$ and $E_{(U-B)}=0.38 \pm$ 0.03 .

The internal precision and accuracy of our photometry (see Paper I) leaves us confident of the moderately metal poor nature assigned to the cluster. The best simultaneous fits in all the CMDs (Figs. 3-5) were obtained for 250 and $320 \mathrm{Myr}$ isochrones $(\log (t)=8.4-8.5$ and $Z=0.004 \pm 0.002)$ at $V-M_{V}=$ $13.95 \pm 0.2$, corresponding to a distance of $d=3.0 \pm 0.3 \mathrm{kpc}$ 


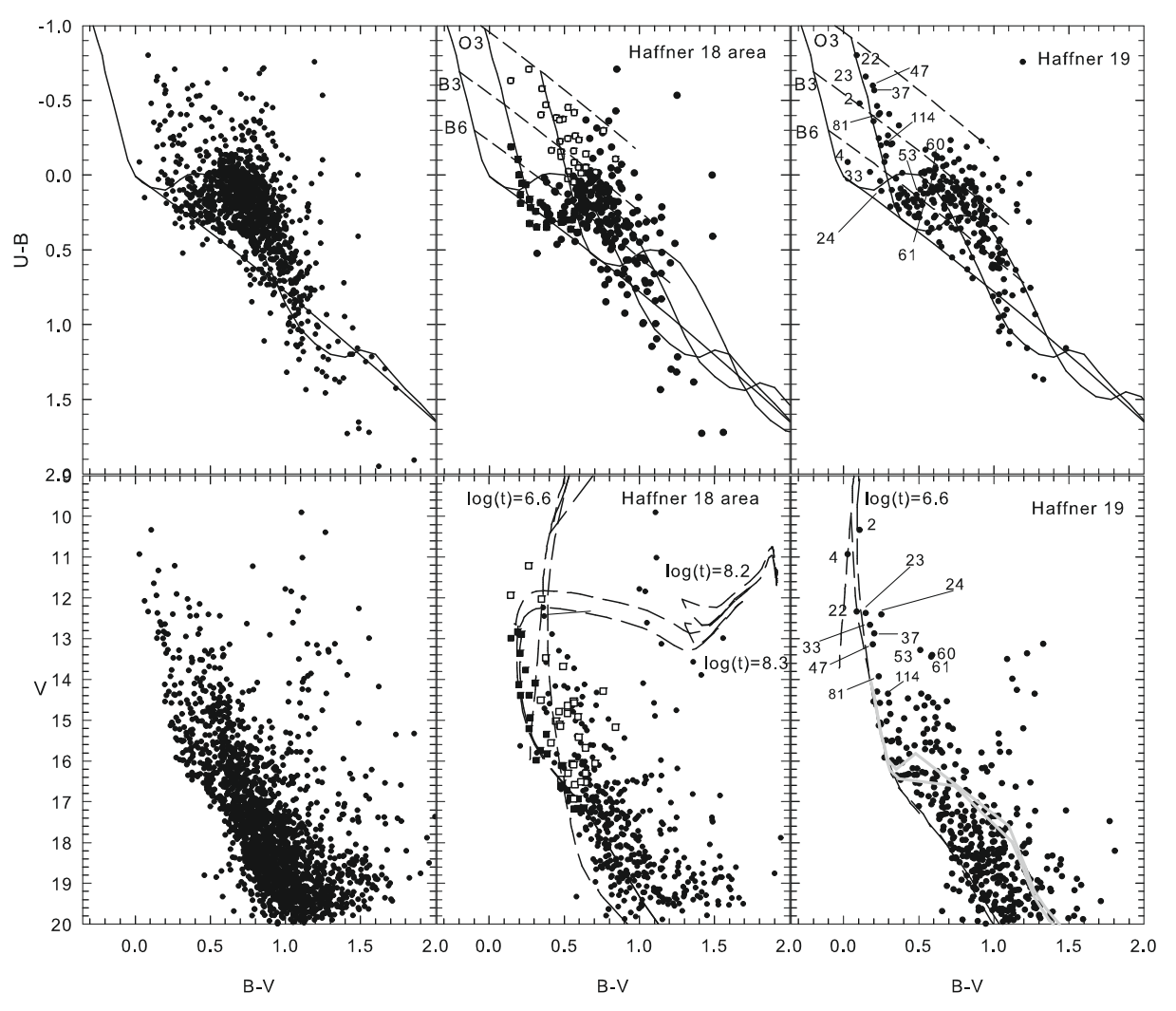

Fig. 6. Upper panels show the TCDs for the entire region (left); Haffner 18 area (Haffner 18(1), filled squares, and Haffner 18(2), open squares) (middle); and Haffner 19 (right). The SKZ (solid line) is shown in its normal position and shifted to fit the respective cluster sequences. The colour excess relations for an O3-, B3-, B6- and A0-type stars are as in Fig. 3. Lower panels show the corresponding CMDs. Long-dashed lines in Haffner 18 area -Haffner 18(1) and Haffner 18(2)- and in Haffner 19 are the isochrone fittings from $\mathrm{G} 00$, labeled $\log (t)$. The right lower panel shows the fitting to Haffner 19 of pre-main sequence isochrones (grey lines, solar metal content) from Siess et al. (2000) for 4 (upper) and 5 (lower) Myr (see text). A number of relevant stars is pointed out in Haffner 19 for a better judgement of their positions in both diagrams. after correcting for visual absorption. This makes Czernik 29 not only metal poor but also relatively young. We draw attention to star \#18. It is well fitted by the red giant loop in the $V$ vs. $B-V$ CMD and is in the giant region of the TCD, making it a possible cluster giant.

The main sequence of Czernik 29 does not shows the broadening seen in Haffner 10 even though their separation is small. However, we note that the field of Haffner 10 is somewhat larger and that variations in the field close to the crossing of two spiral arms are expected.

As previously mentioned, Pietrukowicz et al. (2006) performed a $B V I$ study of Haffner 10 and Czernik 29. In the case of Haffner 10 , our values of $E_{B-V}$, abundance, and age agree with theirs and our distance determination is not far from their distance lower limit. Regarding Czernik 29, the analysis of Pietrukowicz et al. (2006) casts some doubt on its reality as a cluster and except for angular size, no other parameter is determined. The analysis (and deeper photometry) presented here confirms that Czernik 29 is a real cluster. Yet, the large difference in distances and the different metal content of Haffner 10 and Czernik 29 exclude their physical association. They are simply randomly aligned.

\subsection{Haffner 18 and Haffner 19}

Haffner 18 and Haffner 19, as the previous clusters, are located in Puppis and form part of the most complex spatial structure analysed in this article. The interaction between stars and gas, the abrupt change of visual absorption (due to the Puppis window) and the proximity to the nearby emission nebulosity associated to NGC 2467 create a confusing panorama. Far and nearby stellar groups with low and high extinction call into question membership assignments (see Moreno-Corral et al. 2005, for further discussion) and parameter determinations. The strong and variable presence of dust is clearly seen in the maps of Schlegel et al. (1998).

To the best of our knowledge, the most detailed attempt to explain these two clusters is that of FitzGerald \& Moffat (1974). From their analysis of photoelectric and photographic $U B V$ photometry and spectral types they found that Haffner 18 and Haffner 19 are young and situate both clusters at a similar distance of about $6.9 \mathrm{kpc}$ (allowing for an error of almost $1 \mathrm{kpc}$ ). Furthermore, FitzGerald \& Moffat (1974) argue that at such a distance both clusters should be part of what they called the $15 \mathrm{kpc}$ spiral arm, now identified as the Norma-Cygnus arm.

Munari \& Carraro (1996) carried out UBVRI CCD photometry in the field of Haffner 19 and spectroscopy of four bright stars in the field. They measured a variable colour excess, spanning approximately $0.5 \mathrm{mag}$, with a mean value of $E_{(B-V)}=0.44$ and derived a distance of $5.1 \mathrm{kpc}$. Moreno-Corral et al. (2002) acquired $U B V R I$ and $u b v y \beta$ photometry in the region of Haffner 19 and confirmed the distance reported by Munari \& Carraro (1996).

Munari et al. (1998) obtained spectroscopy of 7 stars and $U B V R I$ CCD photometry of the field of Haffner 18, deriving a mean colour excess $E_{(B-V)}=0.62$, an age of $2 \times 10^{6} \mathrm{yr}$ and a distance of $6.3 \mathrm{kpc}$ from the Sun. Moreno-Corral et al. (2005) reported spectral classification for some tens of stars in the field of Haffner 18 and located this object at a distance of $d=8.4 \mathrm{kpc}$ with an error of $1.2 \mathrm{kpc}$. Such differences in the parameters derived for the same object and using similar techniques illustrate the complexity of the analysis and highlight the need for deeper observations.

Figure 6, left upper and lower panels, depicts the overall TCD and CMD of the entire Haffner 18 and 19 area surveyed in this work. The TCD shows a large number of non-uniformly reddened blue bright $\mathrm{OB}$ stars, as expected from the presence 


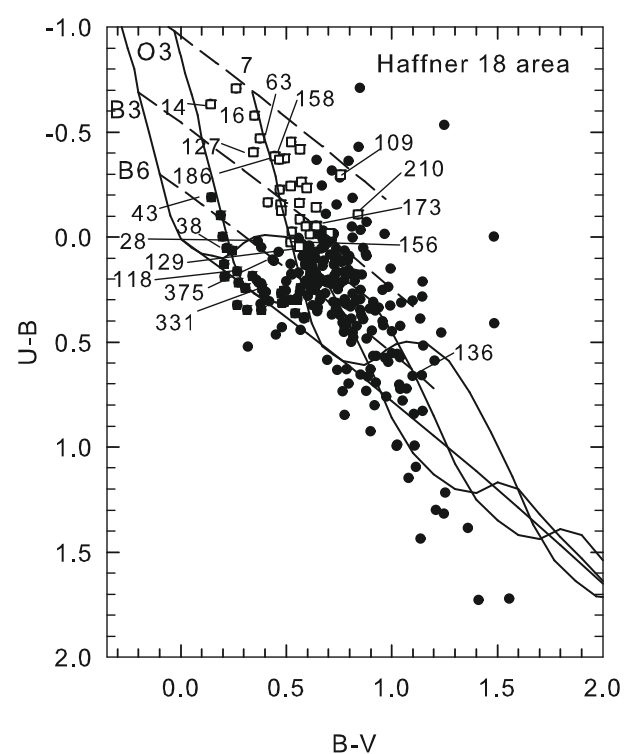

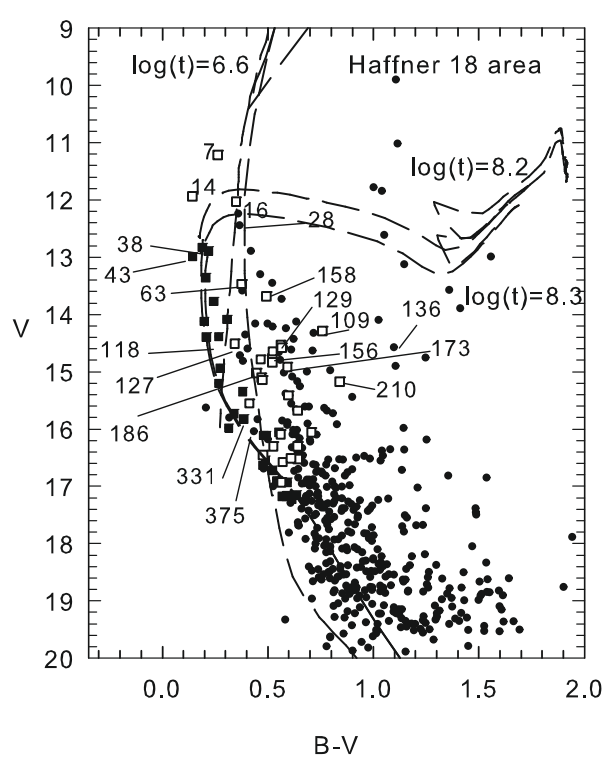

Fig. 7. Left: enlargement of the upper middle panel, $U-B / B-V$, in Fig. 6. Right: enlargement of the lower middle panel, $V / B-V \mathrm{CMD}$, from Fig. 6. Some relevant stars are pointed out for a simultaneous judgement of their positions in both diagrams. Symbols and lines have the same meaning as in Fig. 6. of a strong emission complex [probably belonging to the Puppis OB2 association (Havlen 1972)] and dust clouds in this Galactic location. In addition, the bulk of the stars appear as a dense clump within the colour ranges $-0.1<U-B<0.4$ and $0.5<B-V<1.0$. The overall CMD shows two clear star sequences well separated for magnitudes brighter than $V \approx 17$.

Besides our photometry we also make use of available spectral types of stars in the field of Haffner 18/19. The spectral classifications and their sources are listed in Table 2.

\subsubsection{Haffner 18}

The TCD and CMD of the Haffner 18 area (upper and lower middle panels in Fig. 6) display two well defined stellar groups. The TCD shows both groups being affected by distinctive amounts of reddening, resulting in an unambiguous identification of their members.

The less reddened sequence (filled squares) in the TCD and CMD is mainly defined by stars with (photometric) types later than B3. The sequence can be followed down to A-F-types, which corresponds to $B-V<0.5$ and $V \sim 16.5$. We will refer to this group as Haffner 18(1). The best superposition of the SKZ was achieved for a mean colour excess $E_{(B-V)}=0.30 \pm 0.02$, with a little dispersion around the mean. Both the $V / B-V \mathrm{CMD}$ in Fig. 6, as well as the $V / V-I C M D$ in Fig. 8, show a broadening of the upper part of this group that may be due to unresolved visual pairs. For a better interpretation of our assertions, we show in Fig. 7 an enlargement of the TCD and CMD of Haffner 18 area, where several stars labeled in our id system allow the reader to verify the coherence between their positions in the TCD and CMD.

The second stellar group, which we call Haffner 18(2), is clearly separated from the first one. Open squares are used to indicate its most discernible elements. Haffner 18(2) is mostly composed of very reddened blue stars that can be traced down to approximately the locus of B3 stars. Afterwards, they merge into the stellar background (also mixing with components of Haffner 18(1)) precluding further analysis. In agreement with earlier works, differential reddening is strong, ranging from $E_{(B-V)} \sim 0.60$ to 1.0. Except for a few of the stars, $E_{(B-V)}=0.60$ is the colour excess of the front-line stars composing this group. This colour excess and range are similar to the ones derived by

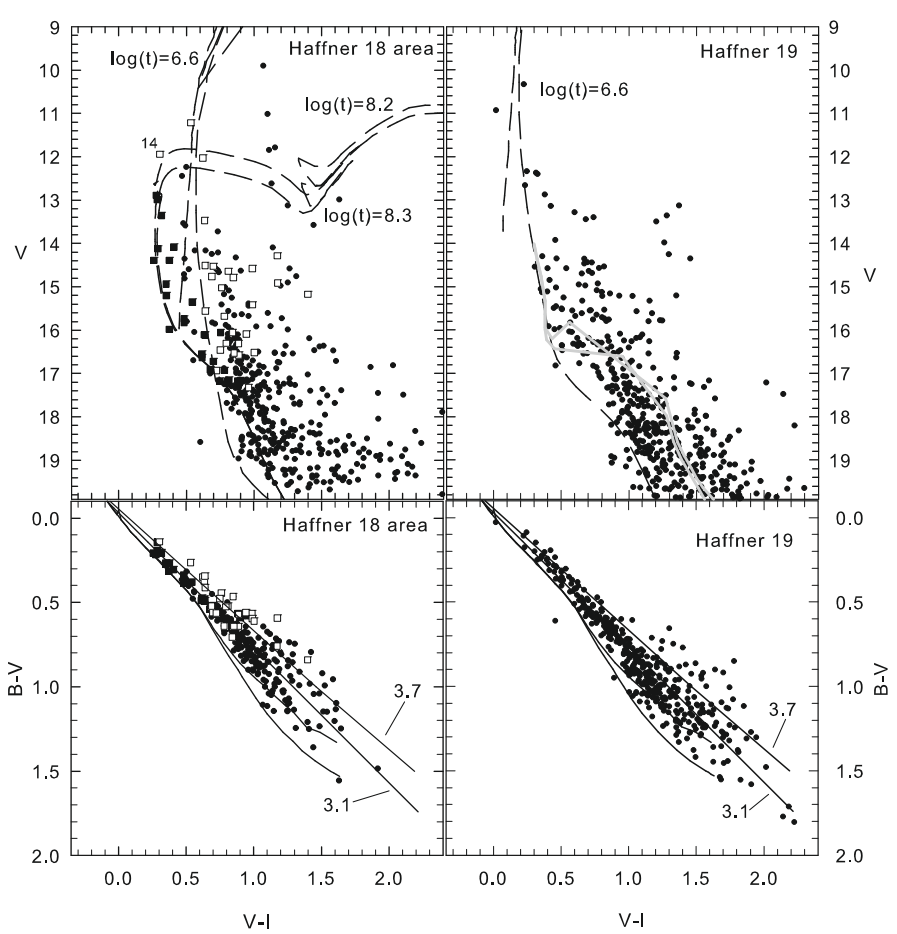

Fig. 8. Left and right upper panels show the $V / V-I$ CMD for Haffner 18 area [Haffner 18(1) and Haffner 18(2)], and Haffner 19 respectively. Symbols and lines have the same meaning as in Fig. 6. Grey lines are the best pre-main sequence fitting from Siess et al. (2000). Left and right lower panels are the respective TCDs, $B-V / V-I$. Symbols have the same meaning as in Fig. 6. Solid lines as in Fig. 4.

Munari et al. (1998). As with the previous group, some of the Haffner 18(2) have published spectral types.

Moreno-Corral et al. (2005) affirm that the extinction law in the direction to Haffner 18 could be assumed to be normal but that NIR photometry would help to settle the issue. The $V-I$ vs. $B-V$ diagrams of both sequences are shown in the lower panels of Fig. 8. The diagram suggests that the extinction law may vary from the normal $R=3.1$ value to the anomalous value of $R=3.7$ (dashed line). Similarly to other regions with anomalous extinction laws (eg. Trumpler 16 or Trumpler 14 Vazquez et al. 1996), 
quez et al.: Binary clusters

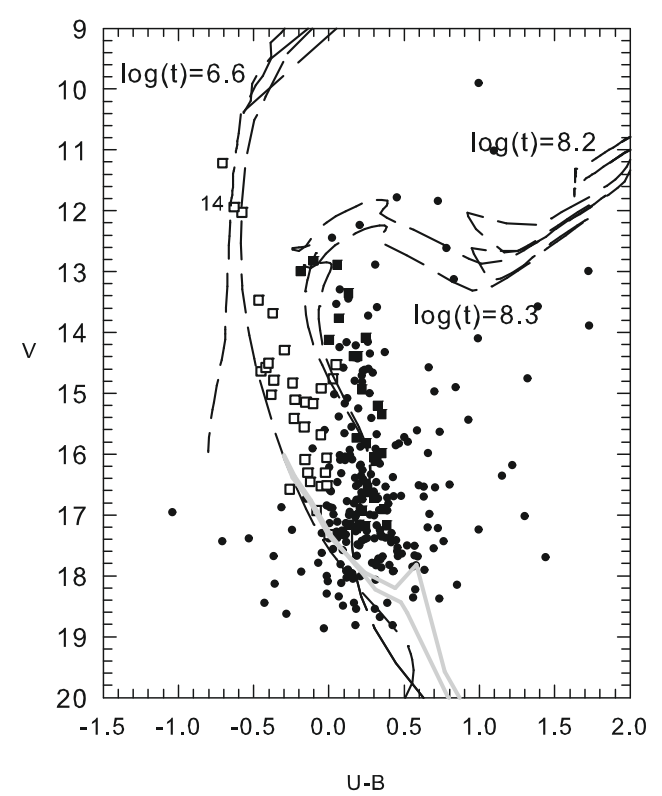

Fig. 9. The $V / U-B$ CMD of Haffner 18(1) and Haffner 18(2); longdashed lines represent the best isochrone $[\log (t)]$ fittings. Grey lines are the solar content pre-main sequence isochrones of 4 (upper) and 5 (lower) Myr from Siess et al. (2000). Symbols as in Fig. 6.

we find stars belonging to both groups and to the field affected by normal and anomalous extinction, as if some of them were strongly affected by the denser interstellar medium while others were not. For some sources, the anomalies will be the result of circum-stellar envelopes, as is the case of star FM3060a-No 14 in our notation- which is surrounded by a ionized hydrogen veil (Moreno-Corral et al. 2005). For others, it will be a result of the recent star forming dust unevenly distributed along the lineof-sight to the region. Despite the evidence of stars affected by anomalous extinction, they are a small fraction. In general terms, reddening toward most stars in the region is characterised by the normal value $R=3.1$.

Adopting $R=3.1$ and $E_{(B-V)}=0.30$, the SKZ CMD fit of Haffner 18(1) yields a distance modulus of $V-M_{V}=$ $14.20 \pm 0.20$, which corresponds to a distance of $4.5 \pm 0.4 \mathrm{kpc}$, after correcting for absorption. The solar composition isochrones (dashed lines) indicate a cluster age between 160 and $200 \mathrm{Myr}$ $(\log (t)=8.2-8.3)$.

Again, $R=3.1$ was adopted for Haffner 18(2) and a colour excess $E_{(B-V)}=0.68$ was deemed representative of the group's reddening. Variable extinction and the youth of the cluster (it contains many early type stars) produce a nearly vertical and fuzzy sequence in the CMD which results in higher than usual ZAMS fitting errors. Therefore, the distance modulus is in the range $17.0<V-M_{V}<17.40$ corresponding to a distance from 9.5 to $11.4 \mathrm{kpc}$ from the Sun. The isochrones best describing the cluster sequence point to an age near $4 \mathrm{Myr}(\log (t)=6.6-6.7)$ which is of the same order as the age reported by Munari et al. (1998). Given the strong internal reddening and the usual change in effective wavelength of the $U$ filter for increasing absorption, the fitting in the $V / U-B$ is not as good as seen in the other CMDs $(V / B-V, V / V-I)$ of Haffner 18(2). However, the fitting goodness in these latter planes secures us a good distance estimate.

Despite this, it is illustrative to see how $U-B$ separates stars of different temperatures as shown in Fig. 9. Haffner 18(1) and Haffner 18(2) appear remarkably well defined and detached from each other. The stars above the isochrone of Haffner 18(2) likely include pre-main sequence objects (PMS; expected at this age)
Table 2. Spectral types of stars in Haffer 18/19.

\begin{tabular}{|c|c|c|c|c|c|c|}
\hline S.Id & FM74 & FM74 & MCCL05 & MCB98 & MC96 & Group \\
\hline 1 & 3084 & G8V & G7V: & & & $\mathrm{H} 18 \mathrm{f}$ \\
\hline 7 & 3081 & O7:k & O7V & O6 & & H18(2) \\
\hline 12 & 3085 & & G0-1V & & & H18f \\
\hline 14 & $3060 a$ & B1Vk & $09.5 \mathrm{~V}$ & B0.5III & & $\mathrm{H} 18(2)$ \\
\hline 16 & 3067 & O9IV:k & O8V & & & H18(2) \\
\hline 19 & $4092 a$ & & G5V: & & & H18f \\
\hline 22 & 4045 & & & & B2V & H19 \\
\hline 28 & 3091 & & A4V & & & $\mathrm{H} 18 \mathrm{f}$ \\
\hline 30 & 4094 & & F5V: & & & $\mathrm{H} 18 \mathrm{f}$ \\
\hline 33 & 3104 & A0 & & & & H19f \\
\hline 37 & 4052 & & & & B2V & H19 \\
\hline 38 & 3092 & A0: & & & & $\mathrm{H} 18(1)$ \\
\hline 42 & 3077 & & K3III & & & H18f \\
\hline 43 & 3074 & & B9/A1V & A0II/III & & H18(1) \\
\hline 47 & 4053 & & & & B3 & H19 \\
\hline 63 & 3070 & & $09.5 \mathrm{~V}$ & & & H18(2) \\
\hline 70 & 3063 & & G8II & & & H18f \\
\hline 74 & 2058 & & G7V & & & $\mathrm{H} 18 \mathrm{f}$ \\
\hline 81 & 4051 & B0Vk & & & & H19 \\
\hline 89 & 4096 & & F7III & & & $\mathrm{H} 18 \mathrm{f}$ \\
\hline 109 & $3072 a$ & & B1V & A4 & & $\mathrm{H} 18(2)$ \\
\hline 114 & 4055 & & & & B7V & H19 \\
\hline 118 & 3059 & & B8V & A2 & & H18(1) \\
\hline 127 & 3078 & & B2V: & & & H18(2) \\
\hline 129 & 2057 & & B6V & & & H18(2) \\
\hline 136 & 3066 & & B1V & & & $\mathrm{H} 18(2) \mathrm{f}$ \\
\hline 156 & 3058 & & B2V & & & $\mathrm{H} 18(2)$ \\
\hline 158 & 4095 & & B4V & & & $\mathrm{H} 18(2)$ \\
\hline 173 & 3057 & & B6V & & & H18(2) \\
\hline 175 & 3060 & & K0II/III & & & H18f \\
\hline 186 & 3082 & & B1V: & & & $\mathrm{H} 18(2)$ \\
\hline 210 & 3073 & B5: & & & & $\mathrm{H} 18(2)$ \\
\hline 331 & 3088 & & F5V: & & & H18(1) \\
\hline 375 & 3079 & & B3V: & & & $\mathrm{H} 18(1) \mathrm{f}$ \\
\hline 398 & 3049 & & & FOII: & & H18f \\
\hline
\end{tabular}

Notes. FM74 indicates the notation and spectral types from FitzGerald \& Moffat (1974). Spectral types in the fourth column (MCCL05) were taken from Moreno-Corral et al. (2005). MCB98 and MC96 are spectral types from Munari et al. (1998) and Munari \& Carraro (1996) respectively. "f" after cluster name is used to indicate the star is a field object.

and MS stars scattered by intracluster reddening and by the presence of unresolved sources.

The colour excess range for Haffner 18(2) from $0.60<$ $E_{(B-V)}<1.0$ implies a mean visual absorption of $A_{V} \approx 1.8$ to $A_{V} \approx 3.0 \mathrm{mag}$, quite low for an object located in the distance range 9.5 to $11.4 \mathrm{kpc}$ along the Galactic plane as shown by the bar joining the two points (see Fig. 15). As discussed in Sect. 5 this is consistent with the fact that the HI layer is not coincident with the formal, $b=0^{\circ}$, Galactic plane.

\subsubsection{Some stars in the field of Haffner 18}

A simple inspection of spectral types in Table 2 shows that one spectral sub-type and/or one luminosity class is a normal difference when comparing observations from different authors for the same star. This is in addition to the internal uncertainty stated by the symbol ":" given by a single researcher. A few stars in Table 2 deserve some words:

\# 14: It has been classified B1Vk, O9.5V or B0.5III (see Table 2) and is surrounded by a compact veil of $\mathrm{H} \alpha$ emission (Moreno-Corral et al. 2005). Like 
Moreno-Corral et al. (2005) we agree that this is a clear sign of a very young object, consistent with the age of Haffner 18(2). This veil affects its colour indices and moves the stars "leftward" in the $U-B / B-V$ plane as if it had $E_{(B-V)}=0.45$. If we remove this value from the observed $B-V=0.14$ and $V=11.94$ we get $(B-V)_{0}=-0.31$, consistent with any of its possible spectral types. Accepting a very anomalous $R$ value of 4.5 , the above mentioned reddening and assuming a mean absolute magnitude $M_{V}=-5$ (in view of its spectral type uncertainty), its distance from the Sun is about $9.5 \mathrm{kpc}$ in agreement with the distance range of Haffner 18(2). The conclusions do not change if we adopt $E_{(B-V)}=0.68$ and $R=3.1$.

\# 28: In Fig. 7 this star (A4V) is not associated with any of the clusters. It is thus a field star.

\# 375: This star has been classified as B3V. However, the colours are not consistent with this classification but indicate a later type $(\sim \mathrm{B} 6)$. Given its location and reddening in the TCD, it is a field star not related to the clusters.

\#136: According to Moreno-Corral et al. (2005) it is a B1V star. A photometric spectral type $b 1$ was given by FM74. However, the position in the $U-B / B-V$ plane is not entirely consistent with the spectral classification. Our photometry gives $B-V=1.09$ and $U-B=0.66$ that, after excess removal, $E_{(B-V)}=0.30$ [if it were in the Haffner 18(1) field] or 0.6 [if it were in Haffner 18(2)] becoming $(B-V)_{0}=0.79$, $(U-B)_{0}=0.40$ or $(B-V)_{0}=0.49$ and $(U-B)_{0}=-0.05$, respectively. Neither solution corresponds to a B1V-type star. The photoelectric $U-B$ photometry from FM 74 and ours differs by more than $0.6 \mathrm{mag}$ and by 0.05 and 0.07 in $B-V$ and $V$ respectively. Munari et al. (1998) photometry did not measure the $U-B$ index but for the rest of indices, $B-V$, $V-R, V-I$ and $V$, the differences are always below 0.07 . We assume that the nearby star 3067 (an O8V-type star) and the small aperture telescope used by FM74 (the $61 \mathrm{~cm}$ at La Silla) may have contributed to contaminating the $U-B$ index. From the position in the TCD, where it looks like a late type star, and the CMD where it is as bright as some other less reddened young objects in Haffner 18(2), we conclude that the spectral type is probably wrong and it is a foreground object.

\subsubsection{Haffner 19}

For Haffner 19 (Fig. 6, right upper and lower panels for the TCD and CMD respectively), only one group of moderately reddened blue stars is noticed in the area. Only a few bright interlopers are present within the established cluster limits.

Although simpler than in the case of Haffner 18, the CMDs (lower panel of Fig. 6, and upper right panel of Fig. 8) easily can be incorrectly interpreted. For example, the brightest stars in these diagrams are not cluster members. Indeed, stars 22 and 23 may be part of Haffner 19 while stars 2, 4, 33 and 24 are field stars. The same applies to stars 53, 60 and 61. Taking special care with the memberships, the SKZ fits yield a colour excess $E_{(B-V)}=0.38 \pm 0.02$ and a distance modulus of $V-M_{V}=15.20 \pm$ 0.25 , corresponding to a distance of $d=6.4 \pm 0.6 \mathrm{kpc}$ after correction for visual absorption. This distance is within the limits given by FitzGerald \& Moffat (1974). It is however $1 \mathrm{kpc}$ closer than the distances obtained by Moreno-Corral et al. (2002) and Munari \& Carraro (1996). As with most very young clusters, the age is not well constrained by post-MS isochrones. Taking into account the lack of any evolved members and the consistency of the isochrone fit (plotted in all CMDs), an age of $4 \mathrm{Myr}(\log (t)=$ 6.6-6.7) is estimated.

Another relevant feature in Haffner 19 is the absence of main sequence stars fainter than $V \sim 16.5$. Given the youth of the cluster, it is interpreted as being due to lower mass members being on the PMS phase. The absolute magnitude at which the MS ends, $M_{V} \sim 0.0$, corresponds to early A-type stars, which is consistent with the estimated age for the cluster. Scaled to the reddening and distance modulus, pre-main sequence isochrones of 4 and $5 \mathrm{Myr}$, from Siess et al. (2000), have been superimposed in the $B / B-V$ and $V / V-I$ planes showing good agreement with the nuclear age derived from Girardi et al. (2000). Later type cluster members are thus redder and brighter than their ZAMS counterparts and are hidden among the field stars in the CMDs. The faint end of the MS is seen in both CMDs, although much more clearly in the $V / V-I$ plane (see Lyra et al. 2006, for a detailed comparison of the use of different colour-magnitude combinations in PMS characterisation). Given the youth of the cluster with objects in the contraction phase it is expected that we will detect anomalies in the extinction law as seen in Fig. 8 (lower panel).

\subsubsection{Haffner 18ab and Haffner 18(1) and Haffner 18(2)}

FitzGerald \& Moffat (1974) consider Haffner 18 as an elongated stellar structure, almost parallel to the galactic plane, composed of two adjacent and indistinguishable clusters which they label Haffner 18a and Haffner 18b. However, they suggest caution by saying that their data did not allow then to decide whether absorbing dust clouds could be responsible for this peculiar elongated morphology. The contour map shown in Fig. 2 reveals a more spherical structure only detectable when all stars down to $V \sim 21$ are taken into account, thus indicating that the influence of dust clouds is important amongst the faintest objects. Our photometry reveals two groups superposed along the line of sight. However, contrarily to FitzGerald \& Moffat (1974), we find them to be at different distances. The closest one, Haffner $18(1)$, is less concentrated than the farther Haffner 18(2).

\subsection{NGC 2383 and NGC 2384}

These objects belong to the constellation of Canis Major at the extreme of an elephant trunk-like structure in the maps of Schlegel et al. (1998). NGC 2383 is a compact cluster as seen in Fig. 1. Approximately $6^{\prime}$ southeast, a handful of bright blue stars appear as a cigar-shaped structure that has been historically identified as the open cluster NGC 2384 (see Fig. 1). Both objects were observed by Vogt \& Moffat (1972) who found them to be separated by more than $1 \mathrm{kpc}$ thus excluding their physical association and later FitzGerald et al. (1979) performed spectral classification for ten star in the fields of both clusters. Subramaniam \& Sagar (1999) obtained BVRI photometry and spectroscopy of both clusters finding that they are very close, only 9 pc from each other. They did however discard a common origin based on the very different ages estimated for both aggregates.

The global TCD and CMD of the surveyed region (upper and lower left panels respectively in Fig. 10) show the presence of a sequence comprising very blue hot stars with $U-B<0.5$ and $B-V<0.54$. The bulk of the stellar distribution is noticeable as a clump in the TCD within $0.5<B-V<1.3$ and $0.0<U-B<$ 0.5 . Its counterpart in the CMD is represented by the notorious stellar distribution that broadens at $V>16$.

The density contour plot (Fig. 2) only reveals the presence of NGC 2383. In all trials using different kernel sizes, NGC 2384 

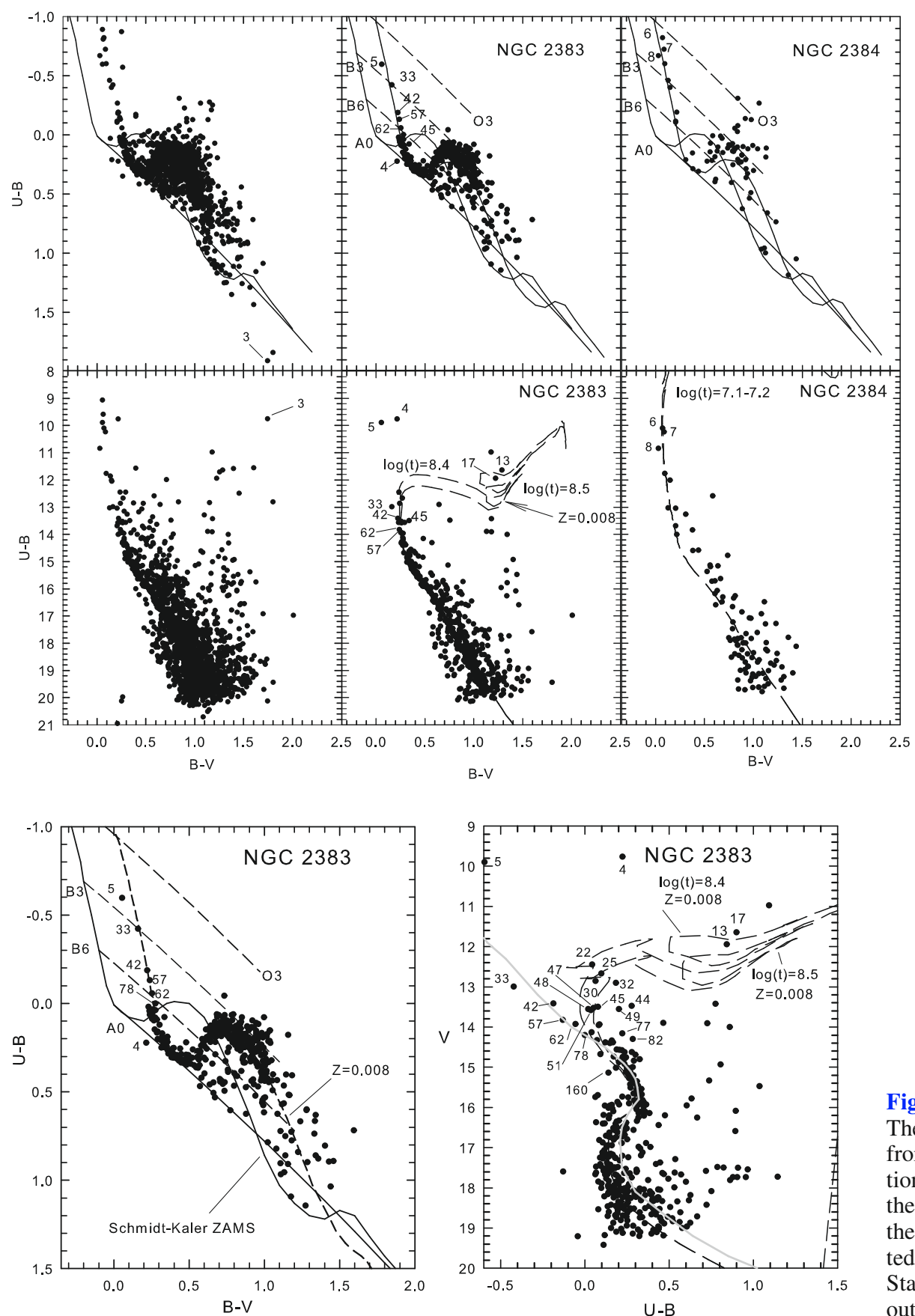

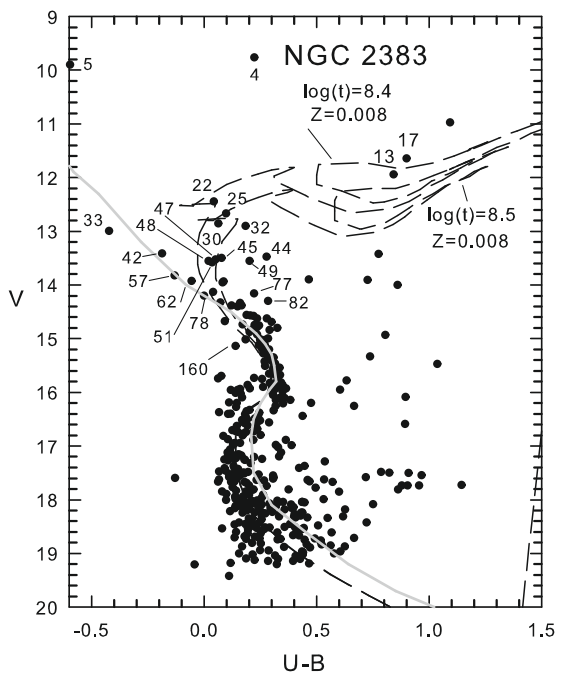

Fig. 10. Upper panels show the TCDs for the entire region (left), NGC 2383 (middle) and NGC 2384 (right). The SKZ is shown in its normal position and shifted to fit the respective cluster sequences. The colour excess relations for O3-, B3-, B6- and A0-type stars are indicated. Lower panels indicate the correspondent CMDs; long-dashed lines show two isochrone $[\log (\mathrm{t})]$ fittings from $\mathrm{G} 00$ at $Z=0.008$ for NGC 2383 and at solar metal content in NGC 2384. Some stars in both clusters are pointed out using our notation see text. always appeared as a marginal handful of bright and hot stars without a faint counterpart adding to the stellar density. As discussed in Sect. 3.1, further analysis assumes that NGC 2384 is contained within a circle comprising the bright stars. The TCDs and CMDs of NGC 2383 and NGC 2384 are shown in Fig. 10 (middle and right panels, respectively).

\subsubsection{NGC 2383}

The sequence of NGC 2383 is very well defined in the photometric diagrams (Fig. 10 middle panels). At a first glance, one is tempted to consider the few dispersed hot B-type stars with $U-B<0.0$ as members of NGC 2383. However, the cluster is only seen from late $\mathrm{B}$ - and A-type stars with $U-B>0.0$ to later types. The TCD of the cluster members is characterised by a slight internal scatter that broadens the sequence and is identifiable down to $B-V \approx 1.0$. In the CMD, NGC 2383 is well detached from the field exhibiting a tight sequence from $13 \simeq V<\simeq 20$. The few hot B-type stars with $U-B<0.0$ that could be taken as members of NGC 2383 in the TCD are all above $V=14$ and are not related to the cluster. Some of these stars (labelled in our figures) are hot according to their location in the TCD, but too faint in the CMD, suggesting no connection with NGC 2383: they are stars 33, 42, 57, 62 and 78 to which we return later.

We notice that the SKZ does not fit well all the stars of NGC 2383 in the TCD. Indeed, as seen in Fig. 10 - upper middle panel -, as in the case of Czernik 29, a close inspection reveals that the solar SKZ does not follow the A-F-type stellar sequence, which appears to be metal deficient. The G00 relation with metallicity $Z=0.008 \pm 0.001$ produces a much better match to the data for $E_{(B-V)}=0.30 \pm 0.05$ as shown in the left panel of Fig. 11. In the right panel of Fig. 11, some stars are 


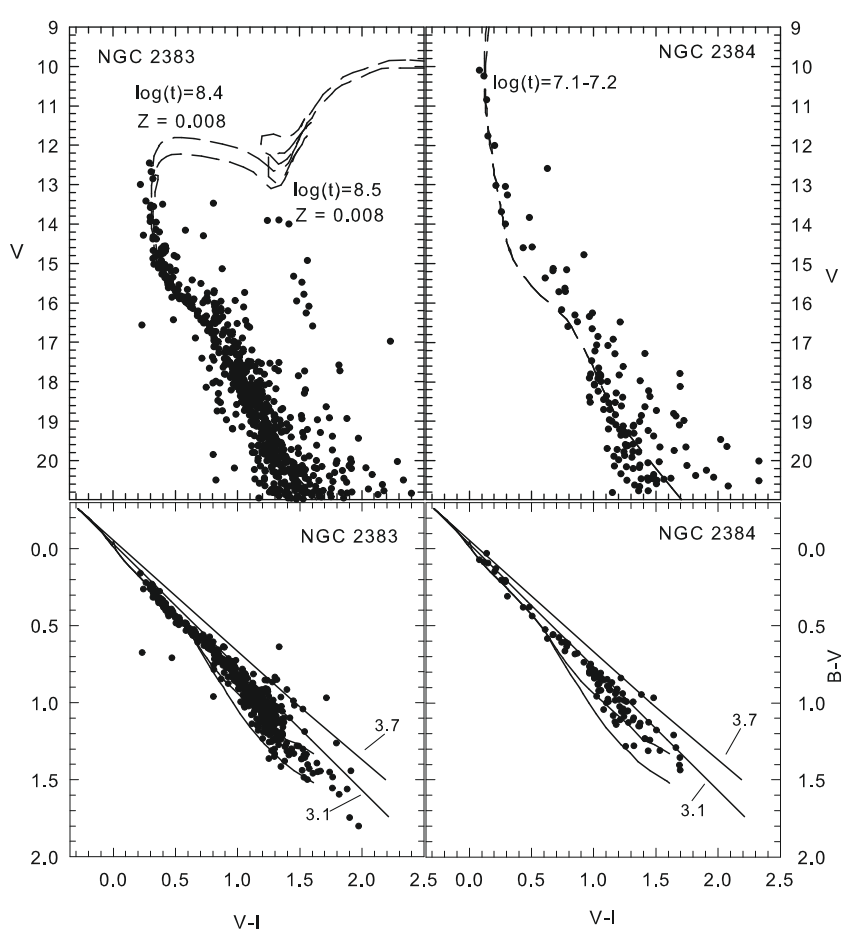

Fig. 12. Upper and lower panels have the same meaning as in Figs. 4 and 8 for the indicated $Z$ content. Lower panels as in Figs. 4 and 8.

indicated for the readeriæ $¡$ s guidance: Nos. 22, 25, 30, 47, 48, 45 and 51 belong to the upper main sequence of NGC 2383 whereas Nos. 4, 5, 44, 49, 77 and 82 are obvious field stars. It is interesting to note that stars $33,42,57,62$ and 78 do not follow the cluster isochrones and may have no relation to NGC 2383. Star 33, in particular, was given a spectral type of A3V by Subramaniam \& Sagar (1999), although we note that its observed colours suggest an earlier spectral type of around B3.

Considering the $V / B-V$, the $V / U-B$ and the $V / V-I$ CMDs simultaneously (Figs. 10-12) we see that the cluster sequence is contaminated by a few interlopers, which only start introducing some spread in the stellar distribution for $15>V$.

As for the extinction law, Fig. 12, lower panel shows that with the exception of a few stars with possible anomalous colours, it is a completely $R=3.1$ law. Adopting a colour excess of $E_{(B-V)}=0.30$, the best simultaneous data fit in all the CMDs yields $V-M_{V}=13.40 \pm 0.25$, which corresponds to a distance $d=3.12 \pm 0.3 \mathrm{kpc}$. The best isochrone matches indicate a cluster age between 250 and $310 \mathrm{Myr}(\log (t)=8.4-8.5)$. These results agree with those of Subramaniam \& Sagar (1999).

\subsubsection{NGC 2384}

NGC 2384 is an peculiar object visible in the hot region of the TCD in Fig. 10 (upper right panel) and in the brightest region of the CMD in the same figure. The right panels of Fig. 12 show the $V / V-I$ and the $B-V / V-I$ diagrams of the cluster. All diagrams show that NGC 2384 is well defined in the bright sector.

As seen in the lower panel of Fig. 12, the absorption law does not show any signs of anomaly. Adopting $R=3.1$, the SKZ fit to NGC 2384 yields $E_{(B-V)}=0.31 \pm 0.03$, with no clues to the cluster's metal content. The distance modulus is determined to be $V-M_{V}=13.35 \pm 0.20$, corresponding to a distance of $d=3.0 \pm 0.3 \mathrm{kpc}$. The age is estimated to be between 12 and $16 \mathrm{Myr}(\log (t)=7.1-7.2)$, not far from the $20 \mathrm{Myr}$ found by Subramaniam \& Sagar (1999).
Table 3. Spectral types in NGC 2383 and NGC 2384.

\begin{tabular}{lrrrr}
\hline \hline Star Id & V\&M72 & S\&S99 & F79 & Group \\
\hline 3 & S8 & M1I & K3III & Out \\
4 & S2 & A6III & A3V & NGC 2383 \\
5 & S1 & A3I & B0III & NGC 2383 \\
6 & S11* & B1.5V & B3IV & NGC 2384 \\
8 & S13* & B1.5V & & NGC 2384 \\
13 & S7 & K3III & & NGC 2383 \\
17 & S9 & K2III & & NGC 2383 \\
33 & S10 & A3V(s) & & NGC 2383 \\
\hline
\end{tabular}

Notes. Column 2 is the Vogt \& Moffat (1972) star identification. S\&S99 refers to spectral types obtained by Subramaniam \& Sagar (1999). The fourth column denotes spectral types from FitzGerald et al. (1979) and the last one is the membership of some of both (or none) of the clusters.

As noticed in Sect. 3.2, the $U-B$ index is useful for separating different groups. Returning to the $V / U-B$ CMD of NGC 2383 in Fig. 11, we can re-interpret the nature of the few hot stars seen in Fig. 10 (upper panel) and which we have already seen are not connected to NGC 2383. Indeed, as discussed in the previous section, the area of NGC 2383 contains two different groups that separate from each other for $U-B<0.0$. The group defined by the broadened sequence starting at $U-B \simeq 0.0$ is NGC 2383. The other, defined by the hottest stars $33,42,57$, 62 and 78, not only do not follow the sequence of NGC 2383 but also coincide with the colour excess, distance and age of the stars in NGC 2384. To test this idea we have superposed in Fig. 11 (right panel) the SKZ (grey solid line) corrected for the distance modulus and colour excess of NGC 2384. The remarkably good fit achieved for the bluest stars with $U-B<0.0$ seen together with NGC 2383 members indicates that stars belonging to/or connected with NGC 2384 are distributed in a larger area including not only the south-east side of NGC 2383 but also partially the region occupied by NGC 2383 itself. Therefore, NGC 2384 appears thus as a sparse group of bright stars projected against (and slightly closer than) the older cluster NGC 2383. In the light of the results obtained for the handful of stars composing NGC 2384 and summarising, this cluster seems more like a sparse young remnant group of a recent star formation episode in front of NGC 2383.

\subsubsection{Some specific stars in both clusters}

As mentioned earlier, Subramaniam \& Sagar (1999) performed spectral classification of 6 stars in NGC 2383 (listed in Table 3). They concluded that star S1 (No. 5 in our notation) is of type A3I. Our photometry (see the TCD in Fig. 10) indicates it is of an early spectral type, more consistent with the B0III classification given by FitzGerald et al. (1979). Star S2 (4 in our notation) has been classified as A6III by Subramaniam \& Sagar (1999) and A3V by FitzGerald et al. (1979). Our diagrams do not allow us to decide on its most probable luminosity class, only that it is related to NGC 2383.

A special case is that of star S8 (No 3 in our list, indicated in Fig. 10, left panels). Following Subramaniam \& Sagar (1999), it is a background M1I supergiant. We agree that this star is not a member of NGC 2383 but it becomes interesting to analyse its location in the Galaxy: Adopting a mean $M_{V}=-5.6 \pm 0.5$ for an M1I-type star and correcting its visual magnitude, $V=9.74$, for the mean of the colour excesses of NGC 2383, $E_{(B-V)}=0.30$, the corrected distance modulus $V_{0}-M_{V}$ is between 13.51 and 14.50, corresponding to distances from 5 to $8 \mathrm{kpc}$, which makes it a 


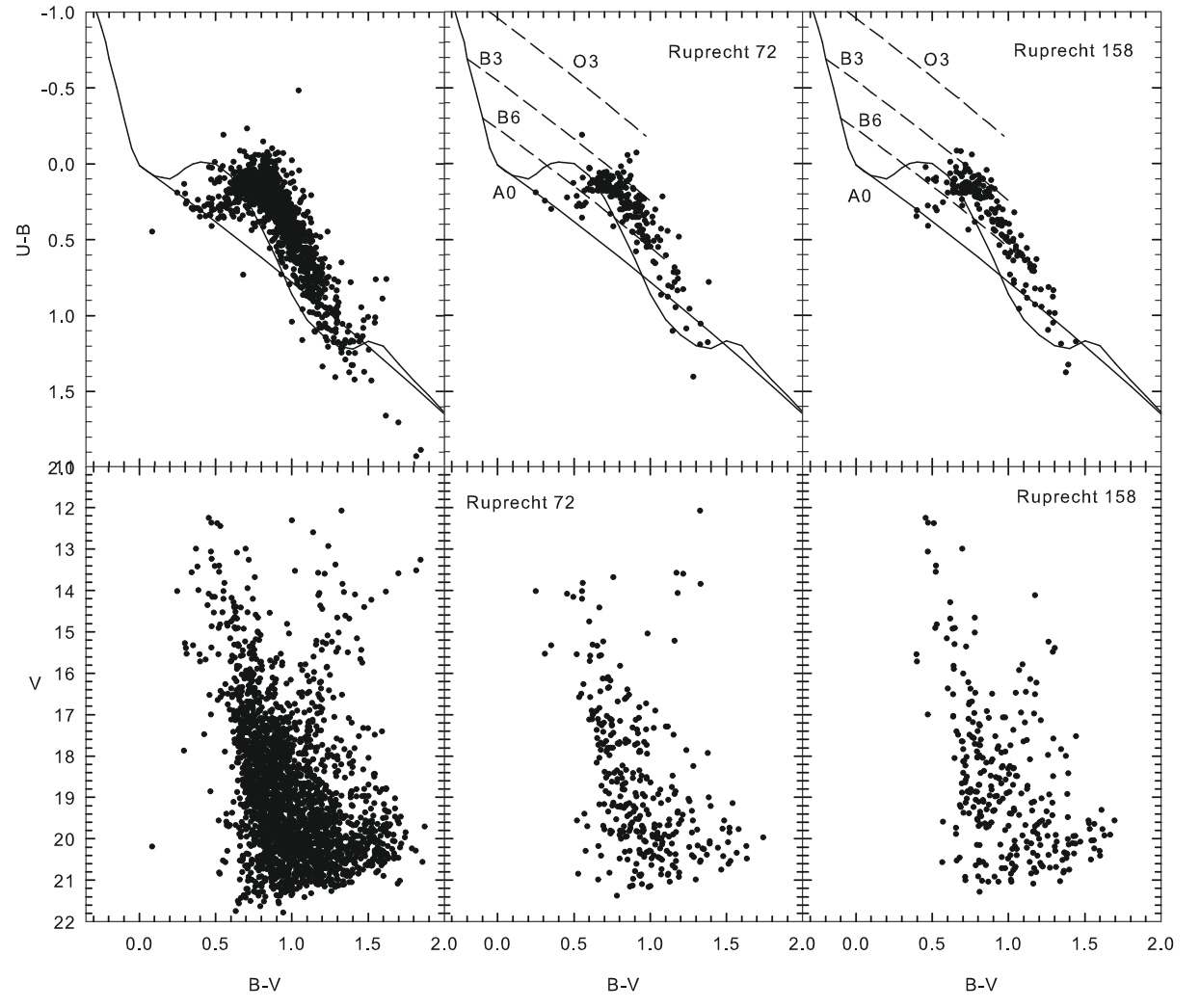

Fig. 13. Upper panels show the TCDs for the entire region (left), Ruprecht 72 (middle) and Ruprecht 158 (right). The SKZ is shown in its normal position. The colour excess relations are indicated. Lower panels have the same meaning as in Figs. 3, 6 and 10. distant background object. Unless the spectral classification from Subramaniam \& Sagar (1999) is wrong, this is a very young object that should not be far from its birthplace, somewhere between the Perseus and Outer arms, as discussed in Sect. 5.

\subsection{Ruprecht 72 and Ruprecht 158.}

The last objects of our study are Ruprecht 72 and Ruprecht 158, located in Puppis. Analysis of the star density (contour plots in Fig. 2) in the area of these supposed open clusters does not reveal any significant stellar enhancement. Visual inspection of the maps in Fig. 1, combined with the density study, suggest that these putative clusters result from a visual impression created by a slight random concentration of a few moderately bright field stars. Nevertheless, an investigation of the parameters listed in the Dias et al. (2002) catalogue was carried out by adopting several radii around the reported cluster centres.

Figure 13 shows the TCD and CMD of the entire field (upper and lower left panels, respectively). It is a typical diagram of a moderately high latitude field containing a significant population of dwarf stars across a range of metallicity together with traces of a moderately young population. This last population is easily seen at $0.6<B-V$ where a knee of late B- and A-type stars appears. Most of these stars are of average brightness and appear mostly above $V \sim 16$. Still, some of them can be tracked down to the faintest magnitudes, indicating that a moderately young population is intercepted all along the line of sight.

If TCDs and CMDs for Ruprecht 72 and Ruprecht 158 (Fig. 13, middle and right panels, respectively) are built assuming a common radius, e.g., $2.5^{\prime}$ around the centres given in the Dias et al. (2002) catalogue, we obtain sparse diagrams resembling those in the left panels representing the total surveyed area. Again, the $V / V-I$ CMDs in the upper panels of Fig. 14 are almost identical. The extinction law checked in the $B-V / V-I$

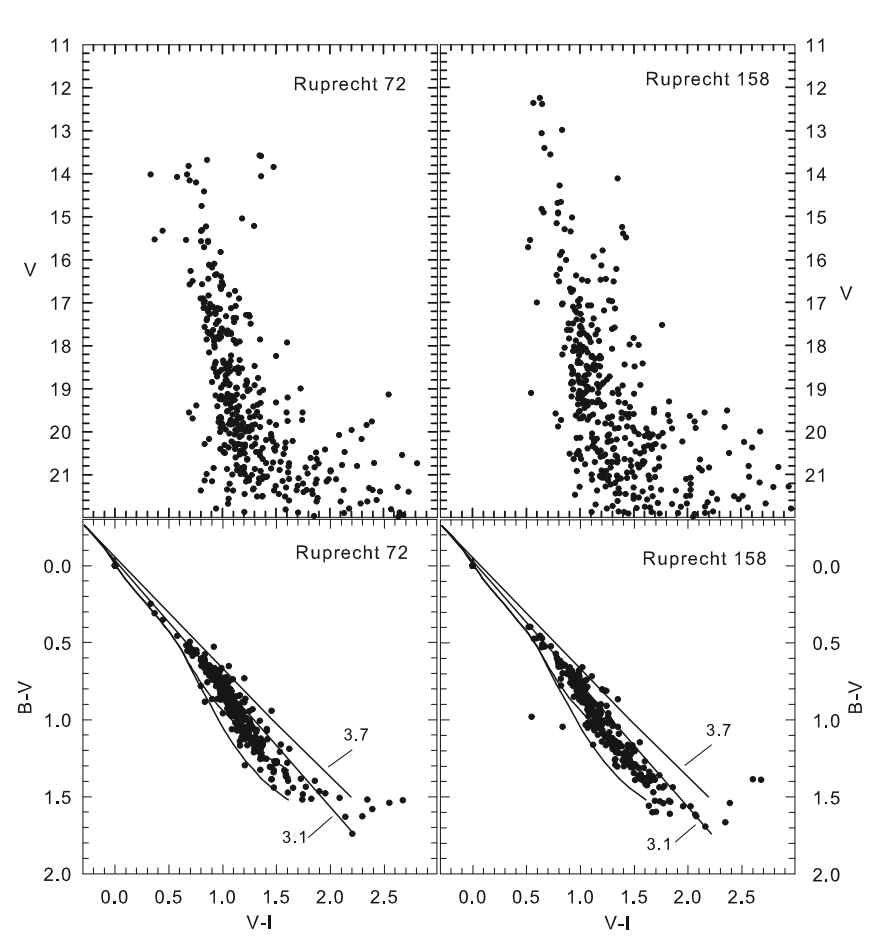

Fig. 14. Upper and lower panels as in Figs. 4, 8 and 12.

diagrams, lower panels in Fig. 14, confirms its normality as asserted in Paper I.

\section{The clusters and spiral structure}

We will now see how the studied clusters fit in the Galactic context. Figure 15, upper panel, is an $X-Y$ projection of the Galactic 


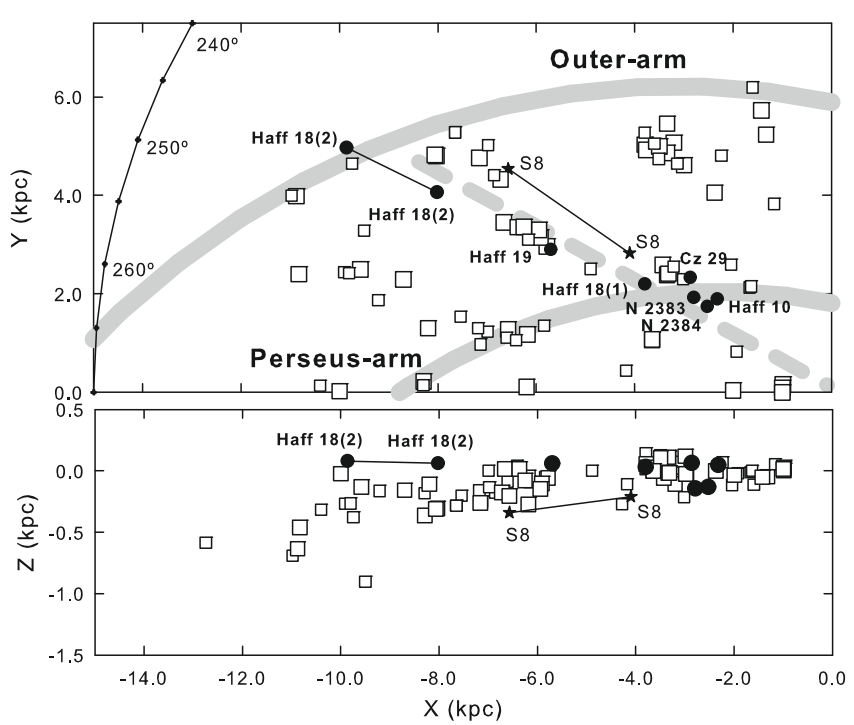

Fig. 15. Upper panel: the X-Y plane with the Sun at 0,0. Open squares are $\mathrm{CO}$ clouds (the largest mean the most massive clouds) in units of $10^{5} M_{\odot}$. Filled circles are the clusters investigated in the present sample. The range of distance found for Haffner 18(2) is indicated by the bar joining the two labeled points. Likewise, stars connected with a line indicate the two possible locations of star S8 in NGC 2383. We also show the path of the Outer- and Perseus-arms according to Vallée (2005) and the axis of the Local arm (long-dashed wide grey line). An angular scale is also drawn. Lower panel: the X-Z plane. Symbols like in the upper panel.

plane ( $X$-axis is negative towards $l=270^{\circ}$ and the $Y$-axis is positive towards the Galaxy anti-centre). The Outer and Perseus arms from Vallée (2005) are drawn for the reader's guidance. In order to compare cluster locations with those of potential star formation complexes, $\mathrm{CO}$ clouds (open squares) with masses higher than $0.5 \times 10^{5} M_{\odot}$ (May et al. 1997, 2008) have been plotted.

Only three clusters have ages that enable them to be potential arm tracers. They are Haffner 18(2), at less than $10 \mathrm{Myr}$ it is a secure component of the Outer arm; NGC 2384, around $15 \mathrm{Myr}$ is at the intersection of the Local arm with Perseus, while Haffner 19, 4 Myr, could be part of the Local arm beyond the crossing of the Perseus arm. The other older clusters, Haffner 18(1), NGC 2383 and Czernik 29 have ages that suggest they are not too far from their birthplaces. In particular, Haffner 18(1) is not far beyond Perseus and may be tracing the Local arm extension out in the Third Galactic Quadrant. Haffner 10 is seen at the position of Perseus but it is probably just passing across given its large age.

The metallicities of NGC 2383 and Czernik 29, $Z=0.008$ and $Z=0.004$, respectively, their similar ages (from 250 to $300 \mathrm{Myr})$ and distances $(d \approx 3.0 \mathrm{kpc})$ indicate a relatively recent star formation event in the last $300 \mathrm{Myr}$ and that this process took place in a less metallic and localised environment. These metallic abundances agree with the expected $[\mathrm{Fe} / \mathrm{H}]$ at such a distance, i.e., $[\mathrm{Fe} / \mathrm{H}] \approx-0.4$ dex to $[\mathrm{Fe} / \mathrm{H}] \approx-0.6$ dex and this puts these two clusters on the plateau already devised through analysis of other objects such as isolated hot stars (Cayrel de Strobel et al. 1997; Daflon \& Cunha 2004), planetary nebula (Costa et al. 2004), Cepheids (Fry \& Carney 1997), etc., though more limited in distance. We cannot argue against or in favour of the existence of a radial metallicity gradient (see Chen et al. 2003; Koen \& Lombard 2007).

Figure 15 also indicates the probable location of the S8 star (No. 3 in our notation) according to the findings of Sect. 4.3.3.
Table 4. Results obtained for the eight clusters.

\begin{tabular}{lrrrrr}
\hline \hline Cl.Name & $V-M_{V}$ & $E_{(B-V)}$ & $\log (t)$ & $\mathrm{d}[\mathrm{kpc}]$ & \multicolumn{1}{c}{$z$} \\
\hline Cz 29 & 13.95 & 0.47 & $8.4-8.5$ & 3.0 & 0.004 \\
& \pm 0.20 & \pm 0.02 & & \pm 0.30 & \pm 0.002 \\
Haff 10 & 14.55 & 0.55 & $9.2-9.4$ & 3.7 & 0.008 \\
& \pm 0.25 & \pm 0.03 & & \pm 0.40 & \pm 0.001 \\
Haff 18(1) & 14.20 & 0.30 & $8.2-8.3$ & 4.5 & - \\
& \pm 0.20 & \pm 0.02 & & \pm 0.40 & \\
Haff 18(2) & $17.0-17.4$ & $0.68^{*}$ & $6.6-6.7$ & $9.5-11.4$ & - \\
& & & & & \\
Haff 19 & 15.20 & 0.38 & $6.7-6.6$ & 6.4 & - \\
& \pm 0.25 & \pm 0.02 & & \pm 0.65 & \\
NGC 2383 & 13.40 & 0.30 & $8.4-8.5$ & 3.1 & 0.008 \\
& \pm 0.25 & \pm 0.05 & & \pm 0.30 & \pm 0.001 \\
NGC 2384 & 13.35 & 0.31 & $7.2-7.1$ & 3.0 & - \\
& \pm 0.20 & \pm 0.03 & & \pm 0.30 & \\
Rup 72 & - & - & - & - & - \\
& - & - & - & - & - \\
Rup 158 & - & - & - & - & - \\
& - & - & - & - & - \\
\hline
\end{tabular}

Notes. Column 1: cluster name; column 2: the $V-M_{V}$ apparent distance modulus and fitting error; column 3: the adopted mean $E_{(B-V}$ colour excess and the range of confidence; $\left({ }^{*}\right.$ see text for the range of $E_{(B-V)}$ in this region); column 4: the range of the $\log (t)$; column 5: cluster distance in kpc and errors; column 6: adopted metal content and confidence range.

Whatever the true distance (based on the single spectral classification estimate from Subramaniam \& Sagar 1999), this is a young star which, at both estimated distance limits, is close to $\mathrm{CO}$ cloud complexes (that is, between the innermost part of the Outer arm and the outermost part of the Perseus arm). It is also possible that $\mathrm{S} 8$ is a member of the extension of the Local arm outward the Third Galactic Quadrant, after its crossing of Perseus. It is remarkable that S8 suffers so little absorption $\left(E_{(B-V)} \sim 0.33\right)$ at such a large distance. Inspecting the maps of Schlegel et al. (1998), NGC 2383 and NGC 2384 are both located near a vast hole in the dust distribution which facilitates observation of distant objects like this star. Such low reddening is also measured toward Haffner 18(2), at $\sim 11 \mathrm{kpc}$, although Haffner 18(2) is almost coincident with the formal Galactic plane and S8 is below it.

Regarding the clusters, only Haffner 18(2) is a plausible member of the Outer arm. According to its Z-height, it is on the northern rim of the HI layer of the Galactic plane: In fact, when looking at Figs. 3, 4 and 9 of Voskes \& Burton (2006), the HI layer at 14 and $16 \mathrm{kpc}$ from the Galactic centre shows evidence of the warp down to $1.5 \mathrm{kpc}$ below the formal Galactic plane and that the northern part of the layer tends to be located at $b=0^{\circ}$. The warp could thus partially explain the low visual absorption toward Haffner 18(2) in spite of its distance.

\section{Comments and conclusions}

The photometric data analysed in this work have allowed us to characterise the observed clusters and to assess their alleged binary nature. Cluster reddenings, distances, ages and in a few cases metallicities were determined and are listed in Table 4. We find that parameters of stellar systems of the type studied here are more reliable when, besides $B V R I$ photometry, $U$ measurements are also available. In particular, $U$ data provide a check of spectral classification and criteria for efficiently separating field from cluster stars. 
Summarising the main results of this investigation:

a) Czernik 29 and Haffner 10 are not a cluster pair in the sense that they do not have a common origin. Analysis of the photometric diagrams has shown that the two objects have different metallicities and ages, ruling out such a possibility.

b) Haffner 18 and Haffner 19 belong to an extraordinarily complex stellar structure where several young star groups are seen along the line of sight. Our deep photometry has allowed us to disentangle two groups in the direction of Haffner 18 naming the nearest one Haffner 18(1), and the farther one Haffner 18(2). They differ in ages and distances: Haffner 18(1) is located at $4.5 \mathrm{kpc}$ and is characterised by a reddening excess $E_{(B-V)}=0.30$. Haffner 18(2) at a larger distance, between 9.5 and $11.4 \mathrm{kpc}$ from the Sun, is composed of young stars. Haffner 19 is relatively far from Haffner 18(1), more than $1.5 \mathrm{kpc}$ away, though affected by similar reddening. This fact and the very different ages precludes a common origin.

c) NGC 2383 and NGC 2384 do not seem to form a pair either. Both groups have similar reddenings and distances but quite different ages. It was found that NGC 2383 is considerably metal-poor with $Z \sim 0.008$. No traces of a density enhancement of faint stars were detected around NGC 2384. Apparently, NGC 2384 is some kind appendix of a dispersed young stellar structure slightly closer than NGC 2383.

d) Ruprecht 72 and Ruprecht 158 are not clusters but slight random fluctuations of the field stellar density.

Of the 5 optically visible double cluster candidates in this region of the Galaxy, we have analysed 4. For one, it has been found that its components were not clusters at all. None of the analysed pairs has qualified as a binary cluster, indicating that, as opposed to the LMC, these systems are rare in the outskirts of the Galaxy. This could mean that:

a) Double cluster lifetimes are short. No significant active star forming regions are seen presently in this sector of the Galaxy, meaning that statistically they would have already decoupled or merged. However, this hypothesis does not seem to be supported by the small number of candidate pairs, 2 , within the known embedded clusters in the same sector of the Galaxy.

b) Double clusters form preferentially in environments different to that of the $3 \mathrm{GQ}$, closer to the environment of the LMC. In that sense, it would be relevant to evaluate the frequency of binary clusters toward denser and more violent regions of the Milky Way, such as the inner Galaxy.

Acknowledgements. R.A.V. wishes to acknowledge the financial support from the IALP (UNLP-CONICET, Argentina) and the PIP No. 5970 from CONICET. A.M. acknowledges support from F.C.T. (Portugal) through grant POCI/CTEAST/57128/2004. A.M. and R.A.V. also acknowledge support from the ESO Visiting Astronomer Programme. The authors are grateful for useful suggestions from an anonymous referee that improved the article.

\section{References}

Aarseth, S. J. 1996, in The Origins, Evolution, and Destinies of Binary Stars in Clusters, ed. E. F. Milone, \& J.-C. Mermilliod, ASP Conf. Ser., 90, 423 Baume, G., Moitinho, A., Giorgi, E. E., Carraro, G., \& Vázquez, R. A. 2004, A\&A, 417, 961
Baume, G., Moitinho, A., Vázquez, R. A. et al. 2006, MNRAS, 367, 1441 Bhatia, R. K. 1990, PASJ, 42, 757

Bica, E., Dutra, C. M., \& Barbuy, B. 2003, A\&A, 397, 177

Blaauw, A. 1963, Baltical Astronomical Data, Univ. of Chicago, 383

Carraro, G., Vázquez, R. A., Moitinho, A., \& Baume, G. 2005, ApJ, 630, L153

Carraro, G., Vázquez, R. A., \& Moitinho, A. 2008, A\&A, 482, 777

Cayrel de Strobel, G., Soubiran, C., Friel, E. D., Ralite, N., \& Francois, P. 1997, A\&AS, 124, 299

Chen, L., Hou, J. L., \& Wang, J. J. 2003, AJ, 125, 1397

Costa, R. D. D., Uchida, M. M. M., \& Maciel, W. J. 2004, A\&A, 423, 199 Cousins, A. W. J. 1978, MNSSA, 37, 62

Cutri, R. M., Skrutskie, M. F., van Dyk, S. et al. 2003, explanatory Supplement to the 2MASS All Sky Data Release

Daflon, S., \& Cunha, K. 2004, ApJ, 617, 1115

de La Fuente Marcos, R. 1997, A\&A, 322, 764

de Oliveira, M. R., Bica, E., \& Dottori, H. 2000, MNRAS, 311, 589

Dean, J. F. Warren, P. R., \& Cousins, A. W. J. 1978, Dean, 183, 569

Dias, W. S., Alessi, B. S., Moitinho, A., \& Lépine, J. R. D. 2002, A\&A, 389, 871

Dieball, A., \& Grebel, E. K. 2000, A\&A, 358, 897

Dieball, A., Müller, H., \& Grebel, E. K. 2002, A\&A, 391, 547

Dutra, C. M., Bica, E., Soares, J., \& Barbuy, B. 2003, A\&A, 400, 533

Efremov, Y. N., \& Sitnik, T. G. 1988, PAZh, 14, 817

FitzGerald, M. P., \& Moffat, A. F. J. 1974, AJ, 79, 873

FitzGerald, M. P., \& Moffat, A. F. J. 1980, PASP, 92, 489

FitzGerald, M. P., Luiken, M., Maitzen, H. M., \& Moffat, A. F. J. 1979, A\&AS, 37,345

Fry, A. M., \& Carney, B. W. 1997, AJ, 113, 1073

Girardi, L., Bressan, A., Bertelli, G., \& Chiosi, C. 2000, A\&AS, 141, 371

Kassis, M., Janes, K. A., Friel, E. D., \& Phelps, R. L. 1997, AJ113, 1723

King, I. 1962, AJ, 67, 471

Koen, C., \& Lombard, F. 2007, MNRAS, 382, 693

Kroupa, P., Aarseth, S., \& Hurley, J. 2001, MNRAS, 321, 699

Landolt, A. U. 1992, AJ, 104, 340

Lyra, W., Moitinho, A., van der Bliek, N. S., \& Alves, J. 2006, A\&A, 453, 101

Martin, N. F., Ibata, R. A., Bellazzini, M. et al. 2004, MNRAS, 348, 12

May, J., Alvarez, H., \& Bronfman, L. 1997, A\&A, 327, 325

May, J., Bronfman, L., Max-Moerbeck, W., \& Olmos, F. 2008, ApJ, submitted

Meynet, G., Mermilliod, J.-C., \& Maeder, A. 1993, A\&AS, 98, 477

Moitinho, A. 2001, A\&A, 370, 436

Moitinho, A., Carraro, G., Baume, G., \& Vazquez, R. A. 2006a, A\&A, 445, 493

Moitinho, A., Vázquez, R. A., Carraro, G. et al. 2006b, MNRAS, 368, L77

Moreno-Corral, M. A., Chavarría-K., C., \& de Lara, E. 2002, Rev. Mex. Astron. Astrofis., 38, 141

Moreno-Corral, M. A., Chavarría-K, C., \& de Lara, E. 2005, Rev. Mex. Astron. Astrofis., 41, 69

Munari, U., \& Carraro, G. 1996, MNRAS, 283, 905

Munari, U., Carraro, G., \& Barbon, R. 1998, MNRAS, 297, 867

Phelps, R. L., \& Janes, K. 1994, ApJS, 90, 31

Pietrukowicz, P., Kaluzny, J., \& Krzeminski, W. 2006, MNRAS, 365, 110

Schlegel, D. J., Finkbeiner, D. P., \& Davis, M. 1998, ApJ, 500, 525

Schmidt-Kaler, T. 1982, Landolt-Börnstein, Group VI, 2b, Stars and Star Clusters (Berlin: Springer), 15

Siess, L., Dufour, E., \& Forestini, M. 2000, A\&A, 358, 593

Stetson, P. B. 1987, PASP, 99, 181

Subramaniam, A., \& Sagar, R. 1999, AJ, 117, 937

Turner, D. G. 1974, AJ, 78, 597

Turner, D. G. 1976, AJ, 81, 97

Vallée, J. P. 2005, AJ, 130, 569

Vazquez, R. A., Baume, G., Feinstein, A., \& Prado, P. 1996, A\&AS, 116, 75

Vázquez, R. A., May, J., Carraro, G. et al. 2008, ApJ, 672, 930

Vogt, N., \& Moffat, A. F. J. 1972, A\&AS, 7, 133

Voskes, T., \& Burton, W. B. 2006, The Warped HI Layer of the Outer Galaxy, M.Sc. Thesis, University of Leiden, under supervision of W. B. Burton. [arXiv:astro-ph/0601653]

Ziyakhanov, R. F., Nuritdinov, S. N., Muminov, M. M., \& Muslimova, Y. C. 2004, Order and Chaos in Stellar and Planetary Systems, Proceedings of the Conference held 17-24 August, 2003 at St. Petersburg State University, Russia. Edited by Gene G. Byrd, Konstantin V. Kholshevnikov, Aleksandr A Myllri, Igor I. Nikiforov and Victor V. Orlov. (San Francisco: Astronomical Society of the Pacific), ASP Conf. Proc., 316, 192 